\title{
The Cauchy Problem for Effectively Hyperbolic Equations
}

\section{(A Standard Type)}

By

\author{
Nobuhisa IWASAKI*
}

\section{§ 0. Introduction}

The previous paper [4] by the author discussed the Cauchy problem for a special type of hyperbolic equations of second order to prove its $C^{+\infty}$-wellposedness. This paper will show that any effectively hyperbolic equation of second order has such a special expression of symbols, namely, that the special type may be regarded as a standard type of effectively hyperbolic equation of second order. Once this proposition has been admitted, the combination with the result of the previous paper could justify, for equations of second order, the assertion that any effectively hyperbolic equation is strongly hyperbolic, namely, $C^{+\infty}$-wellposed independent of lower terms.

Our discussion starts from the following premise.

The principal part $p_{2}$ of an operator of second order has a form that

$$
p_{2}=\xi_{0}^{2}-f\left(x_{0}, x, \xi\right) \text {. }
$$

$p_{2}$ is hyperbolic with respect to the direction $d x_{0}$, that is, $f$ is a non negative function.

$p_{2}$ is effectively hyperbolic, that is, the fundamental matrix of $p_{2}$ has real non zero eigenvalues at the singular points of characteristics of $p_{2}$.

And $f$ is strictly positive outside a bounded set of $x$ variable as

Received April 8, 1983.

* Research Institute for Mathematical Sciences, Kyoto University, Kyoto 606, Japan. 
an additional assumption.

Our purpose is to find an infinitely differentiable function $\Lambda=$ $\Lambda\left(x_{0}, x, \xi\right)$ of homogeneous order 1 in $\xi$ at a neighborhood of each fixed $x_{0}$ variable such that

$$
\begin{aligned}
& p_{2}=\xi_{0}^{2}-\Lambda^{2}-b_{2}, \\
& \left\{\xi_{0}-\Lambda, b_{2}\right\}+c b_{2}=0
\end{aligned}
$$

with another infinitely differentiable $c\left(x_{0}, x, \xi\right)$ of homogeneous order 0 in $\xi$,

$$
b_{2} \geq 0
$$

and

$$
\left(\partial / \partial x_{0}\right) \Lambda>0
$$

at $A=0$ and $b_{2}=0$, where $\{$,$\} stands for the Poisson bracket.$

The geometric consideration of the assumptions leads us to the existence of a function $\Lambda_{1}=\Lambda_{1}\left(x_{0}, x, \xi\right)$ of homogeneous order 1 in $\xi$ such that

$$
\left\{\xi_{0}-\Lambda_{1}, f-\Lambda_{1}^{2}\right\}=L[\nabla f, f: \nabla f, f] \quad \text { (bilinear in }(\nabla f, f) \text { ) }
$$

and

$$
\Lambda_{1}=0 \text { and }\left(\partial / \partial x_{0}\right) \Lambda_{1}>0
$$

at $\Sigma=\{f=0\}$.

In order to construct the function $\Lambda$ as a small perturbation of $\Lambda_{1}$, it suffices to solve the quasilinear equation of $\phi$ that

$$
\left\{\xi_{0}-\left(\Lambda_{1}+\phi\right), f-\left(\Lambda_{1}+\phi\right)^{2}\right\}+c\left[f-\left(\Lambda_{1}+\phi\right)^{2}\right]=0 .
$$

This equation is rather simple. In fact it seems that the NashMoser implicit function theorem is applicable to solve it. It is, however, found difficult to give a priori a function $c$ to apply directly the Nash's theorem to the equation. This difficulty is caused by the linear part with the principal part of a singular vector field, while it is not caused if it suffices to obtain only a finitely differentiable solution of any order. But it is avoidable by means of some simple modification of approximate solutions. Therefore our method to find a solution of the quasilinear equation is merely imitative of the Nash's method, though the proof will be given here. We refered the method to Hörmander [1]. 


\section{§1. Preliminary Study}

Let $\sigma^{1}$ be the canonical two form $\sum d \xi^{j} \wedge d x_{j}$ on $T^{*} \boldsymbol{R}^{n+1}$. For the symbol $p_{2}$, the Hamilton vector field $h$ is defined by $\sigma^{1}(u, h)=\nabla p_{2}(u)$ and the fundamental (Hamilton) matrix $\mathscr{F}$ by $\sigma^{1}(u, \mathscr{F} v)=Q(u, v)$, where $\nabla p_{2}$ is the differential of $p_{2}$ and $Q(u, v)=\left\langle u, \nabla^{2} p_{2} v\right\rangle$ is a quadratic form defined by the Hesse matrix. If $J_{1}$ is defined by $\sigma^{1}\left(u, J_{1} f\right)=$ $f(u)$, then $h=J_{1} \nabla p_{2}$ and $\mathscr{F}=J_{1} \nabla^{2} p_{2}$. We assume that the Hesse matrix is extended by the second order derivatives of $p_{2}$ in the fixed canonical coordinate also outside the singular points of $p_{2}$. Then $h$ and $\mathscr{F}$ may be defined globally on $T^{*} \boldsymbol{R}^{n+1}$.

According to V. Ya Ivriǐ and V. M. Petkov [3] or L. Hörmander [2], the hyperbolicity of $p_{2}$ implies that the fundamental matrix $\mathscr{F}$ has no eigenvalue except for pure real or pure imaginary ones at the singular points of characteristics of $p_{2}$. Moreover non zero real eigenvalues are isolated and they consist of only a pair of eigenvalues, the absolute values of which are same, if they exist. The assumption that $p_{2}$ is effectively hyperbolic means, by definition, the existence of such eigenvalues on the singular points of characteristics. We denote them by $\alpha>0$ and $-\alpha$. Then it is guaranteed that $\alpha$ is extended to an infinitely differentiable function at a neighborhood of the singular points of characteristics as $\alpha$ and $-\alpha$ keep being eigenvalues of $\mathscr{F}$ and real, by virtue of the facts that $\alpha$ and $-\alpha$ are isolated and that $\mathscr{F}$ is a real matrix. Since the fundamental matrix $\mathscr{F}$ is independent of the variable $\xi_{0}, \alpha$ is also independent of the variable $\xi_{0}$ and of homogeneous order 1 in $\xi$. The eigenvector $v_{+}$corresponding to $\alpha$ is also chosen as an infinitely differentiable function in $\left(x_{0}, x, \xi\right)$ at a neighborhood of the singular points of characteristics. It may be normalized as

$$
v_{+}=2^{-1}\left(\partial / \partial \xi_{0}\right)+\alpha^{-1}\left(\partial / \partial x_{0}\right)+v_{2}(\partial / \partial X),
$$

where $X=(x, \xi)$. Then $v_{2}$ satisfies that

$$
\alpha^{-1} f_{x_{0} x_{0}}+\left\langle f_{X x_{0}}, v_{2}\right\rangle=2^{-1} \alpha
$$

and

$$
\alpha^{-1} J_{1} f_{X x_{0}}+J_{1} f_{X X} v_{2}=-\alpha v_{2}
$$

We now extend uniformly positive $\alpha$ and $v_{2}$ as infinitely differentiable 
functions on the whole space to put

$$
\begin{aligned}
\xi_{0}-\Lambda_{1} & =v_{+}\left(p_{2}\right) \\
& =\xi_{0}-f_{x_{0}} \alpha^{-1}-f_{X} v_{2} .
\end{aligned}
$$

Then it holds that

$$
\left\{\xi_{0}-\Lambda_{1}, f-\Lambda_{1}^{2}\right\}=L[\nabla f, f: \nabla f, f] .
$$

Remark on Notations. $L\left[X_{1}: X_{2}: \cdots\right]$ means that it is a multi-linear combination of each component $X_{j}$ with coefficients of infinitely differentiable functions.

In fact $\nabla \Lambda_{1}$ satisfies that

$$
\begin{aligned}
\Lambda_{1 x_{0}} & =\alpha^{-1} f_{x_{0} x_{0}}+f_{x_{0} X} \gamma_{2}+L[\nabla f] \\
& =2^{-1} \alpha+L[\nabla f, f]
\end{aligned}
$$

and

$$
\begin{aligned}
J_{1} \Lambda_{1 X} & =J_{1} f_{X x_{0}} \alpha^{-1}+J_{1} f_{X X} v_{2}+L[\nabla f] \\
& =-\alpha v_{2}+L[\nabla f, f] .
\end{aligned}
$$

Therefore we obtain that

$$
\begin{aligned}
& \left\{\xi_{0}-\Lambda_{1}, f-\Lambda_{1}^{2}\right\}=f_{x_{0}}-\left\{\Lambda_{1}, f\right\}-2 \Lambda_{1} \Lambda_{1 x_{0}} \\
& \quad=f_{x_{0}}+\alpha\left\langle f_{X}, v_{2}\right\rangle-\Lambda_{1} \alpha+L[\nabla f, f: \nabla f, f] \\
& \quad=L[\nabla f, f: \nabla f, f],
\end{aligned}
$$

because $\Lambda_{1}=L[\nabla f],\left\{\Lambda_{1}, f\right\}=\left\langle J_{1} \Lambda_{1 X}, f_{X}\right\rangle$ and $f_{x_{0}}+\alpha\left\langle f_{X}, v_{2}\right\rangle=\alpha \Lambda_{1}$, by definition. Moreover it is clear that

$$
\left(\partial / \partial x_{0}\right) \Lambda_{1}=2^{-1} \alpha>0
$$

at the points such that $f=0$.

In order to attain our purpose, we shall show the following local theorem.

Theorem 1. There exist infinitely differentiable functions $\phi$ and $c$ in $\left(x_{0}, x, \xi\right)$ such that they satisfy the quasilinear equation

$$
\left\{\xi_{0}-\left(\Lambda_{1}+\phi\right), f-\left(\Lambda_{1}+\phi\right)^{2}\right\}+c\left[f-\left(\Lambda_{1}+\phi\right)^{2}\right]=0
$$

and the inequality

$$
\left(\partial / \partial x_{0}\right)\left(\Lambda_{1}+\phi\right)>0
$$


at a neighborhood of the zero points $\sum$ of $f$ and that

$$
\Lambda_{1}+\phi=0
$$

at $\Sigma$.

In fact it is extended to a global theorem in $(x, \xi)$ by means of an ingenious cut off function annihilating $\Lambda=\Lambda_{1}+\phi$ outside a neighborhood of $\Sigma$.

Corollary. For any fixed $x_{0}^{\prime}$, there exists an infinitely differentiable function $\Lambda$ in $\left(x_{0}, x, \xi\right)$ of homogeneous order 1 such that

$$
\left\{\xi_{0}-\Lambda, f-\Lambda^{2}\right\}+c\left[f-\Lambda^{2}\right]=0
$$

on $I \times \boldsymbol{R}^{2 n}$, where $I$ is an open interval including $x_{0}^{\prime}$ and $c$ is also an infinitely differentiable function, and that

$$
\Lambda=0 \text { and }\left(\partial / \partial x_{0}\right) \Lambda \neq 0
$$

at $\Sigma$.

Proof. Let $\rho$ be a monotone decreasing and infinitely differentiable function in a parameter $t$ such that $\rho=1$ if $t \leq 1,1>\rho>0$ if $1<t<3$ and $\rho=0$ if $t \geq 3$. We define $\rho_{\varepsilon}$ as

$$
\rho_{\varepsilon}\left(x_{0}, x, \xi\right)=\rho\left(f|\xi|^{-2} \varepsilon^{-1}\right) \text {. }
$$

If $\varepsilon$ is sufficiently small, then the support of $\rho_{\varepsilon}$ is included in the neighborhood $\Omega$ of $\Sigma$ where the quasilinear equation holds at Theorem 1. We may assume that $f-\Lambda^{2} \geq 0$ on the neighborhood $\Omega$, replacing it to smaller one if necessary, where $\Lambda$ is $\Lambda$ at Theorem 1 .

Let us find another function $\chi_{\varepsilon}$ such that

$$
\left.\left[\chi_{\varepsilon}-\rho\left(6 \Lambda^{2}|\xi|^{-2} \varepsilon^{-1}\right)\right]\right|_{x_{0}=x_{0}}=0
$$

and

$$
\begin{aligned}
\Lambda^{2}\left\{\xi_{0}, \chi_{\varepsilon}^{2}\right\} & +\Lambda\left\{\chi_{\varepsilon}, f\right\}+\left(1-\chi_{\varepsilon}^{2}\right) c f+\chi_{\varepsilon}\left(1-\chi_{\varepsilon}\right)\{\Lambda, f\} \\
& -\left(1-\chi_{\varepsilon}^{2}\right)\left\{\xi_{0}, f\right\}=0
\end{aligned}
$$

at a neighborhood of the intersection of the sets $\left\{x_{0}=x_{0}^{\prime}\right\},\{\varepsilon / 12 \leq$ $\left.\Lambda^{2}|\xi|^{-2} \leq \varepsilon / 3\right\}$ and $\left\{f|\xi|^{-2} \leq 3 \varepsilon\right\}$,

$$
\begin{aligned}
& \chi_{\varepsilon}=1 \text { at } \Lambda^{2}|\xi|^{-2} \leq \varepsilon / 12 \text { and } f|\xi|^{-2} \leq 3 \varepsilon \\
& \chi_{\varepsilon}<1 \text { at } \Lambda^{2}|\xi|^{-2} \geq \varepsilon / 3
\end{aligned}
$$


and $\operatorname{supp} \chi_{\varepsilon}$ is included in $\left\{\Lambda^{2}|\xi|^{-2} \leq \varepsilon / 2\right\}$. Such a function $\chi_{\varepsilon}$ exists on $I \times \boldsymbol{R}^{2 n}$ if the interval $I$ including $x_{0}^{\prime}$ is sufficiently small.

So we put

$$
\Lambda^{\sim}=\chi_{\varepsilon} \rho_{\varepsilon} \Lambda
$$

Then $f-\Lambda^{2}$ satisfies that

$$
\left\{\xi_{0}-\Lambda^{\sim}, f-\Lambda^{2}\right\}+c\left[f-\Lambda^{2}\right]=0
$$

at the open set of $I \times \boldsymbol{R}^{2 n}$ that

$$
f|\xi|^{-2}<\varepsilon \text { and } \Lambda^{2}|\xi|^{-2}<\varepsilon / 3 .
$$

On the other hand we conclude that $f-\Lambda^{2}>0$ at the points that $f|\xi|^{-2} \geq \varepsilon$ or $\Lambda^{2}|\xi|^{-2} \geq \varepsilon / 3$ if $\varepsilon$ is sufficiently small. Therefore it is divisible by $f-\Lambda^{2}$ there in the class of infinitely differentiable functions. We get the conclusion that with another function $c^{\prime}$,

$$
\left\{\xi_{0}-\Lambda^{\sim}, f-\Lambda^{\sim 2}\right\}+c^{\prime}\left[f-\Lambda^{2}\right]=0
$$

on $I \times \boldsymbol{R}^{2 n}$.

$$
\text { q. e.d. }
$$

Since the domain, on which the quasilinear equation should be solved, is a neighborhood of the zero points of $f$, we may change the function $f$ to any form outside it. So we cut off the function $f$ as follows to make the apparent influence of the outside $f$ to the inside as small as possible.

Let us define two functions $\theta(\sigma, t)$ and $\Theta(\sigma, t)$ as

$$
\theta(\sigma, t)=\rho(t / \sigma)
$$

and

$$
\Theta(\sigma, t)=\int_{0}^{t} \theta(\sigma, s) d s
$$

with $\rho$ defined in the previous proof. Moreover let $\phi_{\nu}\left(x_{0}, x, \xi\right)$ denote the solution of the equation that

$$
\left(\partial / \partial x_{0}\right) \psi_{\nu}-\left\{\Lambda_{1}, \psi_{\nu}\right\}=2 \theta\left(\nu^{2}|\xi|^{2}, f\right) \Lambda_{1}\left(\partial / \partial x_{0}\right) \Lambda_{1}
$$

and

$$
\phi_{\nu}=0 \text { at } \Lambda_{1}=0
$$

at a fixed neighborhood of the zero points of $f$, which includes the support of $\theta\left(\varepsilon^{2}|\xi|^{2}, f\right)$ for a positive $\varepsilon$. Then we define $\Lambda_{\theta}^{(2)}$ and $\Lambda_{1-\theta}^{(2)}$ as 


$$
\Lambda_{\theta}^{(2)}=\theta\left(\varepsilon^{2}|\xi|^{2}, f\right) \phi_{\nu} \quad(\geq 0)
$$

and

$$
\Lambda_{1-\theta}^{(2)}=\Lambda_{1}^{2}-\Lambda_{\theta}^{(2)} \quad(\geq 0) .
$$

The modified function $f_{\nu}$ with a parameter $\nu \leq \varepsilon$ is defined as

$$
f_{\nu}=\Theta\left(\nu^{2}|\xi|^{2}, f\right)+\Lambda_{1-\theta}^{(2)} \text {. }
$$

Then it holds that

$$
\begin{aligned}
\left\{\xi_{0}-\Lambda_{1}, f_{\nu}-\Lambda_{1}^{2}\right\} & =\theta\left(\nu^{2}|\xi|^{2}, f\right)\left\{\xi_{0}-\Lambda_{1}, f-\Lambda_{1}^{2}\right\} \\
& -\left\{\Lambda_{1},|\xi|\right\} \nu^{2}(\partial / \partial \sigma) \Theta\left(\nu^{2}|\xi|^{2}, f\right) \\
& -\left\{\xi_{0}-\Lambda_{1}, \theta\left(\varepsilon^{2}|\xi|^{2}, f\right)\right\} \phi_{\nu},
\end{aligned}
$$

and that at a neighborhood of the zero points of $f$

$$
f_{\nu}=f \text {. }
$$

There exists a constant $C$ independent of $\nu$ for $j=0,1$ and 2 such that

$$
\left.|| \xi\right|^{-2+j / 2} \nabla^{j} \Theta\left(\nu^{2}|\xi|^{2}, f\right) \mid \leq C \nu^{2-j}
$$

and

$$
|| \xi !^{-2+j / 2} \nabla^{j} \psi_{\nu} \mid \leq C \nu^{2-j} \text { on } \Omega
$$

so that

$$
\left.|| \xi\right|^{-2+j / 2} \nabla^{j} \Lambda_{\theta}^{(2)} \mid \leq C \nu^{2-j}
$$

Therefore

$$
\left.|| \xi\right|^{-2+j / 2} \nabla^{j}\left(f_{\nu}-\Lambda_{1}^{2}\right) \mid \leq C \nu^{2-j}
$$

and

$$
\left.|| \xi\right|^{-2}\left\{\Lambda_{1-\theta}^{(2)}, \Lambda_{1}\right\} \mid \leq C_{\nu}
$$

so that

$$
\left.|| \xi\right|^{-2}\left\{f_{\nu}, \Lambda_{1}\right\} \mid \leq C_{\nu}
$$

because

$$
\Lambda_{1-\theta}^{(2)}=\Lambda_{1}^{2}-\Lambda_{\theta}^{(2)} \text {. }
$$

Hence it holds for $j=0,1$ and 2 and for

$$
g_{0}=\left\{\xi_{0}-\Lambda_{1}, f_{\nu}-\Lambda_{1}^{2}\right\}
$$

that

$$
\left.|| \xi\right|^{-2+j / 2} \nabla^{j} g_{0} \mid \leq C \nu^{2-j}
$$


Here we use the fact that

$$
|\nabla f|^{2} \leq C f|\xi|
$$

deduced from the non-negativity of $f$.

Remark. The index $\nu$ of $f_{\nu}$ will be omitted in the rest.

\section{§2. A Quasilinear Equation}

The equation, which should be solved, is

$$
\Phi(\phi)-c \Psi(\phi)=0
$$

where

$$
\begin{aligned}
\Phi(\phi) & =-\left\{\xi_{0}-\left(\Lambda_{1}+\phi\right), f-\left(\Lambda_{1}+\phi\right)^{2}\right\} \\
& =2\left(\Lambda_{1}+\phi\right)\left(\partial / \partial x_{0}\right)\left(\Lambda_{1}+\phi\right)-\left\{f, \Lambda_{1}+\phi\right\}-\left(\partial / \partial x_{0}\right) f
\end{aligned}
$$

and

$$
\Psi(\phi)=f-\left(\Lambda_{1}+\phi\right)^{2} .
$$

The function $c$ can not be given a priori, so that we take the Fréchet derivative of the equation in $\phi$ and $c$ under the assumption that $c$ were independent of $\dot{\phi}$. Then we get

$$
\begin{aligned}
& \Phi(\phi+v)-(c+d) \Psi(\phi+v) \\
&= \Phi(\phi)-c \Psi(\phi) \\
&+\Phi_{1}(\phi) v-c \Psi_{1}(\phi) \tau-d \Psi(\phi) \\
&+\Phi_{2}(v)-(c+d) \Psi_{2}(v)-d \Psi_{1}(\phi) v
\end{aligned}
$$

where

$$
\begin{aligned}
& \Phi_{1}(\phi) v=2\left(\Lambda_{1}+\phi\right)\left(\partial / \partial x_{0}\right) v-\{f, v\}+2\left[\left(\partial / \partial x_{0}\right)\left(\Lambda_{1}+\phi\right)\right] v \\
& \Psi_{1}(\phi) v=-2\left(\Lambda_{1}+\phi\right) v \\
& \Phi_{2}(v)=2 v\left(\partial / \partial x_{0}\right) v
\end{aligned}
$$

and

$$
\Psi_{2}(v)=-v^{2} .
$$

A right inverse of the linear part

$$
\left[\Phi_{1}(\phi)-c \Psi_{1}(\phi)\right] v-d \Psi(\phi)
$$

should be obtained for the Nash's method to apply to the quasilinear equation. The difficulty to obtain it occurs from the fact that the principal part of the linear operator is a singular vector field. In fact 
it is impossible in general to obtain an exact right inverse as an operator from infinitely differentiable functions to themselves. The non negativity of the function $f$ and the positivity of $\left(\partial / \partial x_{0}\right)\left(\Lambda_{1}+\phi\right)$ ease the difficulty. We are able only to get approximate ones as follows.

Proposition 2.1. Let $m_{0}$ be a fixed positive integer. If the bounded norms of $\phi, c$ and their derivatives of first order are less than sufficiently small fixed positive constants depending on $m_{0}$ and if the functions $\phi$ satisfy some restrictive conditions (See Remark), then there exist two operators $R_{0}(\phi, c)$ and $R_{1}(\phi, c)$ from the space of infinitely differentiable functions of homogeneous order 0 to the space of infinitely differentiable functions of homogeneous order 0 and 1 , respectively, such that the equality

$$
\left[\Phi_{1}(\phi)-c \Psi_{1}(\phi)\right] R_{1}(\phi, c) g-\phi R_{0}(\phi, c) g=g,
$$

holds on a conic neighborhood of the zero points of $f$ independent of $\phi$ and $c$, where $\phi$ is a sum of two non negative functions and a positive parameter $\kappa$,

$$
\phi=\phi_{0}+\phi_{1}^{2}+\kappa|\xi|^{2},
$$

such that $\phi_{0}$ is the solution of the Cauchy problem

$$
-\left\{\xi_{0}-\left(\Lambda_{1}+\phi\right), \phi_{0}\right\}-c \psi_{0}=0
$$

and

$$
\psi_{0}=f \quad \text { at } \Lambda_{1}+\phi=0,
$$

and that $\phi_{1}$ is also the solution of the equation

$$
-\left\{\xi_{0}-\left(\Lambda_{1}+\phi\right),\left(\Lambda_{1}+\phi\right) \phi_{1}\right\}-c\left(\Lambda_{1}+\phi\right) \psi_{1}=\Phi(\phi)-c \Psi(\phi) .
$$

They satisfy the estimates that

$$
\left\|R_{j}(\phi, c) g\right\|_{m} \leq C_{m}\left[\|g\|_{m}+\|g\|_{0}\left(\|\phi\|_{m+1}+\|c\|_{m}+\|\phi\|_{m}+1\right)\right]
$$

for any non negative integer $m$ less than a fixed integer $m_{0}$, and $j=0$ and 1 , with respect to the norms of the spaces of Hölder continuous functions.

Moreover if the functions $\phi$ satisfy the inequality with a constant $C^{\sim}$ independent of $\kappa$ that

$$
|\xi||\nabla \phi|^{2}+|\phi|^{2} \leq C^{\sim}\left(f+\kappa|\xi|^{2}\right),
$$

then the estimates (2.1) hold for any non negative integer $m$.

Remark. $\left\|\psi_{0}\right\|_{2}$ should be bounded by a constant independent of $\phi$ 
in order to keep the estimates (2.1) for any non negative integer. If function $\phi$ form as

$$
\phi=\rho_{3 \varepsilon} \phi^{\prime}
$$

with $\rho_{3 \varepsilon}\left(\varepsilon=\theta^{-2}\right)$ defined at Section 1 and with the functions $\phi^{\prime}$ satisfying that

$$
\left\|\phi^{\prime}\right\|_{a} \leq C_{a} \delta \theta^{2 a-4}
$$

then there exists a constant $\theta_{0}$, which may depend on $\delta$, such that Proposition 2. 1 holds for any $\phi$ with $\theta \geq \theta_{0}$.

We shall apply Proposition 2.1 only to such a type of functions $\phi$.

Remark on Notations. The Hölder continuous functions of order $\alpha>0$ mean here the functions $g$ that, for the maximum integer $m$ such that $m<\alpha$, the $m$-th derivatives of $g$ are Hölder continuous of order $\alpha-m$ in the usual sense. The norms for them will be extended in a natural way to the homogeneous functions in the variable $\xi$ on any open set (conic in $\xi$ ) of $\boldsymbol{R} \times T^{*} \boldsymbol{R}^{n}$. They are here denoted by $\|\cdot\|_{\alpha}$ and the spaces by $\boldsymbol{H}^{\alpha}$.

Proposition 2.2. Let $\psi$ be a solution of the equation

$$
-\left\{\xi_{0}-\Lambda, \Lambda \psi\right\}-c \Lambda \psi=g
$$

where

$$
\Lambda=\Lambda_{1}+\phi \text {. }
$$

If $\|\phi\|_{2}$ and $\|c\|_{1}$ are uniformly bounded, then for any non negative a, it satisfies the estimate

$$
\|\phi\|_{a} \leq C_{a}\left(\|g\|_{a}+\|g\|_{1}\left(\|\phi\|_{a+1}+1\right)+\|g\|_{0}\left(\|c\|_{a}+1\right)\right) \quad \text { if } a \geq 1
$$

and

$$
\|\phi\|_{a} \leq C_{a}\|g\|_{a} \quad \text { if } 0 \leq a \leq 1,
$$

on a neighborhood of the zero points of $f$ independent of $\phi$ such that $\|\phi\|_{1}$ is sufficiently small.

We now show how to construct asymptotic solutions of $\phi$ and $c$. We start from

$$
\phi_{0}=0
$$

and 


$$
c_{0}=0 \text {. }
$$

So we denote

$$
h_{0}=\Phi(0) \text {. }
$$

$h_{0}$ is divided into two parts such that

$$
h_{0}=h_{0}^{(0)}+h_{0}^{(1)} \text {. }
$$

We shall later show how to divide it. Let us put

$$
h_{n}=\Phi\left(\phi_{n}\right)-c_{n} \Psi\left(\phi_{n}\right)
$$

assuming that $\phi_{n}$ and $c_{n}$ are already given. Then it holds that

$$
\begin{aligned}
& h_{n+1}=h_{n} \\
& \quad+\Phi_{1}\left(\phi_{n}\right) w_{n}^{\tilde{n}}-c_{n} \Psi_{1}\left(\phi_{n}\right) w_{n}^{\tilde{}} \\
& \quad-d_{n}\left[\Psi\left(\phi_{n}\right)+\Psi_{1}\left(\phi_{n}\right) w_{n}^{\tilde{n}}+\Psi_{2}\left(w_{n}^{\tilde{n}}\right)\right] \\
& \quad+\Phi_{2}\left(w_{n}^{\tilde{n}}\right)-c_{n} \Psi_{2}\left(w_{n}^{\tilde{n}}\right),
\end{aligned}
$$

where

$$
\begin{aligned}
& \phi_{j}=\rho_{3 \varepsilon} \phi_{j}^{\prime} \quad(j=n, n+1), \\
& \phi_{n+1}^{\prime}=\phi_{n}^{\prime}+w_{n}, \\
& w_{n}^{\sim}=\rho_{3 \varepsilon} w_{n}
\end{aligned}
$$

and

$$
c_{n+1}=c_{n}+d_{n} \text {. }
$$

We now assume that $w_{n}$ is a solution of the equation

$$
\Phi_{1}\left(\phi_{n}^{\tilde{n}}\right) w_{n}-c_{n}^{\sim} \Psi_{1}\left(\phi_{n}^{\tilde{n}}\right) w_{n}-d_{n} \phi_{n}=g_{n},
$$

where

$$
\phi_{n}^{\tilde{n}}=\rho_{3 \varepsilon} S_{\theta(\theta+n)} \phi_{n}^{\prime}
$$

and

$$
c_{n}=S_{\theta(\theta+n)} c_{n}
$$

Remark on Notations. $S_{\theta}$ is a so-called smoothing operator. An explanation about its properties will be given later.

According to Proposition 2.1, $w_{n}$ may be given by

$$
w_{n}=R_{1}\left(\phi_{n}^{\tilde{n}}, c_{n}^{\tilde{n}}\right) g_{n}
$$

if $d_{n}$ is defined by

$$
d_{n}=R_{0}\left(\phi_{n}^{\tilde{n}}, c_{n}^{\tilde{n}}\right) g_{n}
$$


and if $\phi_{n}$ is $\phi$ with $\kappa=\kappa_{n}=\theta^{1-\alpha}(\theta+n)^{1-\alpha}$ at Proposition 2.1. Then we have that

$$
h_{n+1}=h_{n}+g_{n}+e_{n}+f_{n+1}-f_{n},
$$

where $e_{n}$ and $f_{n}$ are defined as follows. $\phi_{n}$ is also divided into the sum of three terms.

$$
\phi_{n}=\phi_{n}^{(0)}+\phi_{n}^{(1)}+\kappa_{n}
$$

$f_{n}$ and $e_{n}$ are defined as

$$
\begin{aligned}
& f_{0}=h_{0}^{(1)}, \\
& f_{n+1}=f_{n}+d_{n} \psi_{n}^{(1)}-\Phi_{1}\left(\phi_{n}^{\tilde{n}}\right)\left(1-\rho_{3 \varepsilon}\right) w_{n}
\end{aligned}
$$

and

$$
\begin{aligned}
e_{n}= & {\left[\Phi_{1}\left(\phi_{n}\right)-\Phi_{1}\left(\phi_{n}^{\tilde{n}}\right)\right] w_{n}^{\tilde{n}}+\Phi_{2}\left(w_{n}^{\tilde{n}}\right) } \\
& -c_{n+1}\left[\left(\Psi_{1}\left(\phi_{n}\right)-\Psi_{1}\left(\phi_{n}^{\tilde{n}}\right)\right) w_{n}^{\tilde{n}}+\Psi_{2}\left(w_{n}^{\tilde{n}}\right)\right] \\
& -\left(c_{n}-c_{n}^{\tilde{n}}+d_{n}\right) \Psi_{1}\left(\phi_{n}^{\tilde{n}}\right) w_{n}^{\tilde{n}} \\
& -d_{n}\left[\Psi\left(\phi_{n}\right)-\Psi\left(\phi_{n}^{\tilde{n}}\right)+\Psi\left(\phi_{n}^{\tilde{n}}\right)-\phi_{n}^{(0)}-\kappa_{n}\right] .
\end{aligned}
$$

It is put as

$$
e_{n}=e_{n}^{(0)}-d_{n}\left[\Psi\left(\phi_{n}^{\tilde{n}}\right)-\phi_{n}^{(0)}\right]
$$

to be convenient to proof. If $n \geq 1$, then we define

$$
\phi_{n}^{(1)}=\left(\Lambda_{1}+\phi_{n}^{\tilde{n}}\right) \phi_{n}^{(2)}+\left(2 \phi_{n}-\phi_{n}^{(2)}\right) \phi_{n}^{(2)}
$$

by using the solution $\phi_{n}^{(2)}$ of the equation

$$
-\left\{\xi_{0}-\left(\Lambda_{1}+\phi_{n}^{\tilde{n}}\right),\left(\Lambda_{1}+\phi_{n}^{\tilde{n}}\right) \phi_{n}^{(2)}\right\}-c_{n}^{\tilde{n}}\left(\Lambda_{1}+\phi_{n}^{\tilde{n}}\right) \phi_{n}^{(2)}=-f_{n} .
$$

From the above definitions, we get

$$
h_{n+1}=\sum_{j=0}^{n} g_{j}+\sum_{j=0}^{n} e_{j}+f_{n+1}+h_{0}-f_{0} .
$$

Our purpose needs to show that the limit of $h_{n+1}$ exists as $n$ tends to infinity and vanishes at a neighborhood of the zero points of $f$. So we put

$$
\begin{aligned}
& f_{0}=h_{0}^{(1)}, \\
& g_{0}=-S_{\theta^{2}} h_{0}^{(0)}, \\
& E_{0}=0, \\
& E_{n+1}=\sum_{j=0}^{n} e_{j}
\end{aligned}
$$

and

$$
g_{n+1}=-\left(S_{\theta(\theta+n \perp 1)}-S_{\theta(\theta+n)}\right)\left(h_{0}^{(0)}+E_{n}\right)-S_{\theta(\theta+n+1)} e_{n} .
$$

Then we get 


$$
\begin{aligned}
h_{n+1} & =\left(1-S_{\theta(\theta+n)}\right)\left(h_{0}^{(0)}+E_{n}\right)+e_{n}+f_{n} \\
& =e_{n}^{(1)}+f_{n},
\end{aligned}
$$

by defining

$$
e_{n}^{(1)}=\left(1-S_{\theta(\theta+n)}\right)\left(h_{0}^{(0)}+E_{n}\right)+e_{n} .
$$

Here it will be shown at the later proof that $e_{n}^{(1)}$ tends to zero and the limit of $f_{n}$ vanishes at the neighborhood of the zero points of $f$. In order to estimate

$$
\phi_{n}^{(3)}=\Psi\left(\dot{\phi}_{n}^{\tilde{n}}\right)-\phi_{n}^{(0)},
$$

we get it found that it is written as

$$
\psi_{n}^{(3)}=\left(\Lambda_{1}+\phi_{n}^{\tilde{n}}\right) \psi_{n}^{(4)}+\left(\psi_{n}^{(4)}\right)^{2}
$$

by the solution $\phi_{n}^{(4)}$ of the equation

$$
\begin{aligned}
- & \left\{\xi_{0}-\left(\Lambda_{1}+\phi_{n}^{\tilde{n}}\right),\left(\Lambda_{1}+\phi_{n}^{\tilde{n}}\right) \phi_{n}^{(4)}\right\}-c_{n}^{\tilde{n}}\left(\Lambda_{1}+\phi_{n}^{\tilde{n}}\right) \phi_{n}^{(4)} \\
& =e_{n-1}^{(1)}-e_{n}^{(2)},
\end{aligned}
$$

where

$$
e_{n}^{(2)}=\Phi\left(\phi_{n}\right)-\Phi\left(\phi_{n}^{\tilde{n}}\right)-\left[c_{n} \Psi\left(\phi_{n}\right)-c_{n}^{\sim} \Psi\left(\phi_{n}^{\tilde{n}}\right)\right]
$$

We prepare three lemmas for the smoothing operator $S_{\theta}$ and the Hölder spaces. (For example, see Hörmander [1].)

Lemma 2.1. The smoothing operator $S_{\theta}$ has the following properties for $a$ and $b$ such that $0 \leq a(b$, resp. $) \leq M .(\theta \geq 1)$

$$
\begin{array}{lll}
\text { 1) } & \left\|S_{\theta} u\right\|_{b} \leq C_{M}\|u\|_{a} & \text { if } b \leq a . \\
\text { 2) } & \left\|S_{\theta} u\right\|_{b} \leq C_{M} \theta^{b-a}\|u\|_{a} & \text { if } a \leq b . \\
\text { 3) } & \left\|u-S_{\theta} u\right\|_{b} \leq C_{M} \theta^{b-a}\|u\|_{a} & \text { if } b \leq a . \\
\text { 4) } & \left\|(d / d \theta) S_{\theta} u\right\|_{b} \leq C_{M} \theta^{b-a-1}|| u \|_{a} .
\end{array}
$$

Lemma 2.2. $u_{\theta}$ are infinitely differentiable functions with a parameter $\theta$ belonging to $[0,+\infty)$. Let $a(j)$ and $b(j)(j=0$ and 1$)$ satisfy that

$$
a(0)<a(1)
$$

and

$$
b(0)<0<b(1) .
$$

We assume the relations between $a, \lambda, a(j)$ and $b(j)$ as 


$$
\lambda b(0)+(1-\lambda) b(1)=0
$$

and

$$
a=\lambda a(0)+(1-\lambda) a(1) .
$$

If it holds for $u_{\theta}$ that

$$
\left\|u_{\theta}\right\|_{a(j)} \leq M_{j} \theta^{b(j)-1}
$$

with respect to the norms of $\boldsymbol{H}^{a(j)}$ and if the constant $a$ is not integer, then the integral

$$
u=\int_{0}^{+\infty} u_{\theta} d \theta
$$

exists in $\boldsymbol{H}^{a}$ and satisfies that

$$
\left\|u_{a}\right\|_{a} \leq C_{a} M_{0}^{\lambda} M_{1}^{(1-\lambda)} .
$$

Lemma 2.3. Let $u_{i}$ be functions belonging to $\boldsymbol{H}^{a(i, j)}(i=0, \cdots, I$ and $j=0, \cdots, J)$. If we define

$$
a(i)=\sum_{j=0}^{J} a(i, j) \lambda_{j}
$$

by $\lambda_{j}$ such that $0 \leq \lambda_{j} \leq 1$ and $\sum_{j} \lambda_{j}=1$, then the functions $u_{i}$ belong to $\boldsymbol{H}^{a^{(i)}}$ and satisfy the estimate

$$
\Pi_{i=0}^{I}\left\|u_{i}\right\|_{a(i)} \leq C \sum_{j=0}^{J} \Pi_{i=0}^{I}\left\|u_{i}\right\|_{a(i, j)} .
$$

We check the convergence of the approximate solutions $g_{0}$ at (1.3), namely, $h_{0}$ is also $L(\nabla f, f: \nabla f, f)$ at a neighborhood of the zero points of $f$ as well as (1.1). Let us define $h_{0}^{(0)}$ as

$$
h_{0}^{(0)}=\rho_{\varepsilon} h_{0}
$$

by means of $\rho_{\varepsilon}$ at (1.2) with $\varepsilon=\theta^{-4}$. Then it holds that

$$
\text { ! } \mid h_{0}^{(0)} \|_{a} \leq C_{m} \theta^{2 a-4}
$$

for $a$ and $\theta$ such that $0 \leq a \leq m<+\infty$ and $\theta \geq \theta_{0}$ if $\theta_{0}$ is a sufficiently large constant but independent of $m$. And $h_{0}^{(1)}=0$ if $f|\xi|^{-2} \leq \theta^{-4}$. Proposition 2.1 for $\phi=0$ implies that

$$
\left\|w_{0}\right\|_{a} \leq C_{m} \theta^{2 a-4} \text {. }
$$

We shall prove the following lemma.

Lemma 2.4. There exists a constant $\delta$ such that for any non negative integer $j$ and for $0 \leq a \leq m$ (integer), 


$$
\left\|w_{j}\right\|_{a} \text { and }\left\|d_{j}\right\|_{a} \leq \delta \theta^{a+\alpha-4}(\theta+j)^{a-\alpha-1}
$$

if $\alpha>2$ and $2 \alpha+1 \leq m$, and if $\theta$ is sufficiently large after $\alpha$ and $m$ are fixed.

Proof. We prove it by induction in $n \geq 0$. We assume that it holds for $j \leq n$ except for $j=0$ and that $\alpha$ is not integer.

$$
\phi_{k}^{\prime}=\sum_{j=0}^{k-1} w_{j}
$$

satisfies it for $0 \leq k \leq n+1$ that

$$
\begin{aligned}
& \left\|\phi_{k}^{\prime}\right\|_{0} \leq\left(\delta \alpha^{-1}+C_{m}\right) \theta^{-4}, \\
& \left\|\phi_{k}^{\prime}\right\|_{a} \leq\left(\delta(a-\alpha)^{-1}+C_{m}\right) \theta^{a+\alpha-4}(\theta+k)^{a-\alpha} \quad \text { if } m \geq a>\alpha
\end{aligned}
$$

and

$$
\left\|\phi_{k}^{\prime}\right\|_{\alpha} \leq\left(C \delta+C_{m}\right) \theta^{2 \alpha-4}
$$

by Lemma 2.2 because $\alpha$ is not integer. Therefore it holds for $0 \leq$ $k \leq n+1$ that

$$
\left\|\phi_{k}^{\prime}\right\|_{a} \leq C_{\hat{\delta}} \theta^{a+\alpha-4}(\theta+k)^{\operatorname{pos}(a-\alpha)} \theta^{-\operatorname{pos}(\alpha-a)}
$$

for $0 \leq a \leq m$, so that

$$
\left\|\phi_{k}\right\|_{a} \leq C_{\delta} \theta^{a+\alpha-4}(\theta+k)^{\operatorname{pos}(a-\alpha)} \theta^{-\operatorname{pos}(\alpha-a)}
$$

for $0 \leq a \leq m$ by virtue of Lemma 2.3 because

$$
\left\|\rho_{3 \varepsilon}\right\|_{a} \leq G_{m} \theta^{2 a-4} .
$$

In the same way

$$
c_{k}=\sum_{j=0}^{k-1} d_{j}
$$

satisfies it for $0 \leq k \leq n+1$ and for $0 \leq a \leq m$ that

$$
\left\|c_{k}\right\|_{a} \leq C_{\delta} \theta^{a+\alpha-4}(\theta+k)^{\operatorname{pos}(a-\alpha)} \theta^{-\operatorname{pos}(\alpha-a)} .
$$

Remark on Notations. $\operatorname{pos}(s)=\max \{\mathrm{s}, 0\}$.

Therefore we conclude it by virtue of Lemma 2.1 that

$$
\begin{aligned}
& \left\|\phi_{\hat{k}}^{\tilde{k}}\right\|_{a} \text { and }\left\|c_{k}^{\tilde{n}}\right\|_{a} \\
& \quad \leq C_{\hat{\delta}} \theta^{a+\alpha-4}(\theta+k)^{\operatorname{pos}(a-\alpha)} \theta^{-\operatorname{pos}(\alpha-a)}
\end{aligned}
$$

for $0 \leq a \leq a_{0}$ and for $0 \leq k \leq n+1$ with any fixed $a_{0}$ and that

$$
\left\|\phi_{k}-\phi_{\hat{k}}\right\|_{a} \text { and }\left\|c_{k}-c_{k}^{\sim}\right\|_{\alpha} \leq C_{\delta} \theta^{a+\alpha-4}(\theta+k)^{a-\alpha}
$$

for $0 \leq a \leq m$ and for $0 \leq k \leq n+1$, where, by definition,

$$
\dot{\phi}_{\tilde{k}}=\rho_{3 E} S_{\theta(\theta+k)} \phi_{k}^{\prime}
$$


and

$$
c_{\bar{k}}=S_{\theta(\theta+k)} c_{k} .
$$

Lemma 2.5. 1) For $0 \leq a \leq m-1$ and for $0 \leq k \leq n$, it holds that

$$
\begin{aligned}
\left\|e_{k}^{(0)}\right\|_{a} & \leq C_{1 \delta} \theta^{a+2 \alpha-6}(\theta+k)^{a-2 \alpha} \\
& \leq C_{1 \delta} \theta^{a+\alpha-4}(\theta+k)^{a-\alpha-2} .
\end{aligned}
$$

2) For $0 \leq a \leq m-1$ and for $0 \leq k \leq n$, it holds that

$$
\left\|\Psi\left(\phi_{k}\right)-\phi_{k}^{(0)}\right\|_{a} \leq C_{2 \delta} \theta^{a+\alpha-3}(\theta+k)^{a+1-\alpha} .
$$

3) For $0 \leq a \leq m-1$ and for $0 \leq k \leq n$, it holds that

$$
\begin{aligned}
\left\|e_{k}\right\|_{a} & \leq C_{3 \delta} \theta^{a+2 \alpha-6}(\theta+k)^{a-2 \alpha} \\
& \leq C_{3 \delta} \theta^{a+\alpha-4}(\theta+k)^{a-\alpha-2},
\end{aligned}
$$

therefore,

$$
\left\|E_{k+1}\right\|_{a} \leq C_{3 \delta}^{\prime} \theta^{a+\alpha-5}(\theta+k+1)^{\operatorname{pos}(a-\alpha)} \theta^{-\operatorname{pos}(\alpha-a)},
$$

where

$$
E_{k+1}=\sum_{j=0}^{k} e_{k} .
$$

4) For $0 \leq a \leq m-1$ and for $0 \leq k \leq n$, it holds that

$$
\left\|e_{k}^{(1)}\right\|_{a} \leq C_{4 \delta} \theta^{a+\alpha-4}(\theta+k)^{a-\alpha} .
$$

5) For $0 \leq a \leq m$ and for $0 \leq k \leq n$, it holds that

$$
\left\|e_{k}^{(2)}\right\|_{a} \leq C_{5 \delta} \theta^{a+\alpha-3}(\theta+k)^{a+1-\alpha} \text {. }
$$

Remark. The constant $C_{j \delta}$ at the above lemma are independent of $n$.

We assume Lemma 2.5. It will be proved after the present proof. 4) of Lemma 2.1,3) of Lemma 2.5 and the estimate for $h_{0}^{(0)}$ imply that, for $0 \leq a \leq a_{0}$,

$$
\begin{aligned}
& \left\|\left(S_{\theta(\theta+n+1)}-S_{\theta(\theta+n)}\right)\left(h_{0}^{(0)}+E_{n}\right)\right\|_{a} \\
& \quad \leq C \theta^{a-\alpha}(\theta+n+1)^{a-\alpha-1}\left(\left\|h_{0}^{(0)}\right\|_{\alpha}+\left\|E_{n}\right\|_{\alpha}\right) \\
& \quad \leq\left(C_{0}+C_{\delta} \theta^{-1}\right) \theta^{a+\alpha-4}(\theta+n+1)^{a-\alpha-1} .
\end{aligned}
$$

Moreover 1) and 2) of Lemma 2.1 and 3) of Lemma 2.5 imply that for $0 \leq a \leq a_{0}$,

$$
\|\left. S_{\theta(\theta+n+1)} e_{n}\right|_{a} \leq C_{\delta} \theta^{a+\alpha-4}(\theta+n+1)^{a-\alpha-2} .
$$

In fact it suffices to take $b=a$, in case that $a \leq \alpha$, and to put $b=a$ and 
$a=\alpha$, in case that $a \geq \alpha$, at Lemma 2.1. Therefore we obtain that, for $0 \leq a \leq a_{0}$

$$
\left\|g_{n+1}\right\|_{a} \leq 2 C_{0} \theta^{a+\alpha-4}(\theta+n+1)^{a-\alpha-1}
$$

if $\theta$ is taken as

$$
C_{\delta} \theta^{-1} \leq C_{0}
$$

Proposition 2.1 applies to the case that $m=m$ or $m=0$, to yield it for

$$
w_{n+1}=R_{1}\left(\dot{\phi}_{n+1}^{\tilde{1}}, \quad \tilde{n+1}\right) g_{n+1}
$$

and

$$
d_{n+1}=R_{0}\left(\phi_{n+1}^{\tilde{n}}, \tilde{c} \tilde{n+1}\right) g_{n+1},
$$

by virtue of the interpolation theory (Lemma 2.3), that for $0 \leq a \leq m$

$$
\left\|w_{n+1}\right\|_{a} \text { and }\left\|d_{n+1}\right\|_{a} \leq\left(C_{0}^{\prime}+C_{\delta} \theta^{-1}\right) \theta^{a+\alpha-4}(\theta+n+1)^{a-\alpha-1} \text {. }
$$

If $\delta$ is defined as

$$
C_{0}^{\prime} \leq \delta / 2
$$

and if the parameter $\theta$ is chosen as sufficiently large as

$$
C_{\delta} \theta^{-1}<\delta / 2 \text {, }
$$

then the conclusion of induction is deduced because the above constant $C_{\delta}$ is independent of $n$. We here remark that the bound of $\left\|\phi_{k}\right\|_{1}$ should be put less than a small constant given beforehand and that it is satisfied by taking $\theta$ large.

q.e.d.

Proof of Lemma 2.5. 1) Let us estimate $\left(\Phi_{1}\left(\phi_{k}\right)-\Phi_{1}\left(\phi_{k}^{\tilde{k}}\right)\right) w_{k}^{\tilde{k}}$, one of the terms of $e_{k}^{(0)}$. For $0 \leq a \leq m-1$, it holds that

$$
\begin{aligned}
& \left\|\left(\Phi_{1}\left(\phi_{k}\right)-\Phi_{1}\left(\phi_{\tilde{k}}^{\tilde{s}}\right)\right) w_{k}^{\tilde{k}}\right\|_{a} \\
& \leq C\left(\left\|\phi_{k}-\phi_{k}^{\tilde{k}}\right\|_{a+1}\left\|w_{k}^{\tilde{k}}\right\|_{0}+\left\|\phi_{k}-\phi_{\tilde{k}} \tilde{\|}\right\|\left\|_{0}\right\| w_{k}^{\tilde{k}} \|_{a+1}\right) \\
& \leq C_{\delta} \theta^{a+2 a-6}(\theta+k)^{a-2 \alpha} \text {, }
\end{aligned}
$$

because we may apply Lemma 2.3 to the first step and the assumption of induction and the estimates for $\phi_{k}-\phi_{\tilde{k}}$ in the previous proof to the second step. The other terms $e_{k}^{(0)}$ will be estimated in the same way. They have rather better estimates than the above.

2) At first, we note that it suffices to get the same type of estimates for $\psi_{k}^{(4)}$ as for $\psi_{k}^{(3)}=\Psi\left(\phi_{k}^{\tilde{k}}\right)-\phi_{k}^{(0)}$ by definition. It is obtained from the ones of the data $e_{k-1}^{(1)}-e_{k}^{(2)}$ because $\phi_{k}^{(4)}$ is a unique solution of the equation at Proposition 2.2 put there as $\dot{\phi}=\phi_{k}^{\tilde{k}}, \quad c=c_{\tilde{k}}^{\tilde{a}}$ and 
$g=e_{k-1}^{(1)}-e_{k}^{(2)}$, and because $\left\|\phi_{k}\right\|_{2}$ is bounded by a constant independent of $\theta$ and $k$. Therefore we show that $e_{k-1}^{(1)}-e_{k}^{(2)}$ have the same type of estimates with another constant $C_{68}$ independent of $n$. We assume 5) of Lemma 2.5 because it is proved independent of the statement 2). The data $g$ for $\psi_{0}^{(4)}$ is $-e_{0}^{(2)}$ so that the estimate holds in this case if $C_{60}>C_{50}$. Now we also assume them valid for integers not exceeding $k<n$ so that the statement 2) is valid. Then the statements 3) and 4) are valid for such integers as proved at their own proofs. Therefore $e_{k}^{(1)}-e_{k+1}^{(2)}$ is bounded as

$$
\left\|e_{k}^{(1)}-e_{k+1}^{(2)}\right\|_{a} \leq\left(2 C_{4 \delta} \theta^{-1}(\theta+k+1)^{-1}+C_{5 \delta}\right) \theta^{a+\alpha-3}(\theta+k+1)^{a+1-\alpha} .
$$

If the constant $C_{6 \delta}$ is given beforehand as

$$
C_{6 \delta}>C_{5 \delta},
$$

then we are able to choose the parameter $\theta$ as sufficiently large as

$$
C_{6 \delta}>\left(2 C_{4 \delta} \theta^{-1}(\theta+k+1)^{-1}+C_{5 \delta}\right)
$$

and this choice is independent of $k$ and also $n$. Therefore, for $0 \leq$ $a \leq m-1$ and for $0 \leq k \leq n$, it holds that

$$
\| e_{k-1}^{(1)}-\left.e_{k}^{(2)}\right|_{a} \leq C_{6 \delta} \theta^{a+\alpha-3}(\theta+k)^{a+1-\alpha} .
$$

We now apply Proposition 2.2 to get the conclusion.

3) We may assume the statements 1) and 2). Then we obtain that

$$
\begin{aligned}
& \left\|d_{k}\left[\Psi\left(\phi_{\tilde{k}}\right)-\phi_{k}^{(0)}\right]\right\|_{a} \\
& \quad \leq C_{\delta} \theta^{a+2 \alpha-7}(\theta+k)^{a-2 \alpha} .
\end{aligned}
$$

Therefore we get the conclusion because $e_{k}$ is the sum of

$$
e_{k}^{(0)} \text { and }-d_{k}\left[\Psi\left(\phi_{\tilde{k}}\right)-\phi_{k}^{(0)}\right] .
$$

The estimates for $E_{k+1}$ are obtained by applying Lemma 2.2 to it as well as the estimates for $\phi_{k}$ and $c_{k}$ if the estimates for $e_{j}$ hold for integers $j$ not exceeding $k \leq n$.

4) It is proved by applying 2) and 3) of Lemma 2.1, putting $b=a$ and $a=\alpha$, to the results 3) of the present lemma.

5) Since the differences are written as

$$
\Phi\left(\phi_{k}\right)-\Phi\left(\phi_{\hat{k}}^{\tilde{k}}\right)=\Phi_{1}\left(\dot{\phi}_{\tilde{k}}\right)\left(\phi_{k}-\phi_{\hat{k}}^{\tilde{k}}\right)+\Phi_{2}\left(\phi_{k}-\phi_{\tilde{k}}\right)
$$

and

$$
\begin{aligned}
& c_{k} \Psi\left(\phi_{k}\right)-c_{\tilde{k}} \Psi\left(\phi_{\tilde{k}}\right) \\
& \quad=\left(c_{k}-c_{\tilde{k}}\right) \Psi\left(\phi_{k}\right)+c_{\tilde{k}} \Psi_{1}\left(\phi_{\tilde{k}}\right)\left(\phi_{k}-\phi_{\tilde{k}}\right)+c_{\tilde{k}} \Psi_{2}\left(\phi_{k}-\phi_{\tilde{k}}\right),
\end{aligned}
$$


it is not difficult to get the conclusion according to the estimates for $\phi_{k}, \phi_{k}^{\tilde{k}}, \phi_{k}-\phi_{\tilde{k}}, c_{k}, c_{k}^{\tilde{k}}$ and $c_{k}-c_{k}$, by virtue of Lemma 2.3. q. e. d.

Lemma 2.4 implies the existence of limits of $\phi_{n}$ and $c_{n}$ in $\boldsymbol{H}^{\alpha}$, which are denoted by $\phi$ and $c$, respectively. They are estimated as for $0 \leq a \leq \alpha$

$$
\|\phi\|_{a} \text { and }\|c\|_{a} \leq C_{\delta} \theta^{2 a-4} \text {. }
$$

The convergences of $\phi_{n}, c_{n}$ and $e_{n}^{(1)}$ assure the one of $f_{n}$, though it can be estimated directly.

The important fact not clarified yet is that the limit $f_{+\infty}$ of $f_{j}$ vanishes at a neighborhood of the zero points of $f$. The function $f_{0}$ vanishes at a neighborhood $\Omega_{0}$ of the zero points of $f$ by definition. We show that the supports of $\phi_{j}^{(1)}$, therefore, $\phi_{j}^{(2)}$ never fill up the whole of such a domain $\Omega_{0}$. By the definition of $f_{0}$ as

$$
f_{0}=h_{0}^{(1)}=\left(1-\rho_{\varepsilon}\right) h_{0}, \quad \varepsilon=\theta^{-4},
$$

we may put

$$
\Omega_{0}=\left\{f|\xi|^{-2} \leq \theta^{-4}\right\} .
$$

Then the function $f_{0}$ vanishes at $\Omega_{0}$. On the other hand we consider spindle-shaped domains $\Omega_{n}(\tau)$ such

$$
\Omega_{n}(\tau)=\left\{\left|\Lambda_{1}+\phi_{n}^{\tilde{n}}\right|+\mu\left|X-X^{\sim}\right|<\tau\right\}
$$

where $\left(x_{0}^{\tilde{0}}, X^{\sim}\right)=\left(x_{0}^{\tilde{0}}, x^{\sim}, \xi^{\sim}\right)$ is a fixed element in the zero points of $f$ and $\left|\xi^{\sim}\right|=1$. Since it holds that

$$
\left\|\dot{\phi}_{n}^{\tilde{n}}\right\|_{a} \leq \sum_{j=0}^{+\infty}\left\|w_{j}^{\tilde{j}}\right\|_{a} \leq C_{1} \theta^{2 a-4},
$$

the flows starting from $\Lambda_{1}+\phi_{n}^{\tilde{n}}=0$ and difined by the vector fields

$$
\mathscr{L}_{n} v=\left(\partial / \partial x_{0}\right) v-\left\{\Lambda_{1}+\phi_{n}^{\tilde{n}}, v\right\}
$$

sweep out the whole of $\Omega_{n}(\tau)$ if $\mu$ is small and if $\theta$ is sufficiently large, namely, $\left\|\phi_{n}^{\sim}\right\|_{1}$ is small. Therefore we can conclude that $v_{n}$ is identically zero on $\Omega_{n}(\tau)$ if $v_{n}$ is a solution of the equation

$$
\mathscr{L}_{n} v_{n}-c_{n}^{\tilde{v} v_{n}}=0 \quad \text { on } \Omega_{n}(\tau)
$$

and

$$
v_{n}=0 \quad \text { at } \Lambda_{1}+\phi_{n} \tilde{n}=0 .
$$

We now fix a constant $\mu$ independent of $n$ keeping the above property and put 


$$
\tau_{0}=\theta^{-3}
$$

Since $\nabla f$ vanishes at $\left(x_{0}^{\sim}, X^{\sim}\right)$,

$$
f \leq C\left(\Lambda_{1}^{2}+\left|X-X^{\sim}\right|^{2}\right)
$$

so that, on $\Omega_{0}\left(\tau_{0}\right)$,

$$
f \leq C \tau_{0}^{2}=C \theta^{-6} \leq C \theta^{-2} \theta^{-4} .
$$

Therefore $f_{0}$ vanishes at $\Omega_{0}\left(\tau_{0}\right)$ if $\theta$ is sufficiently large. We put

$$
\tau_{n}=\tau_{0}-\sum_{j=0}^{n}\left\|w_{j}\right\|_{0} .
$$

Then it is bounded below such that

$$
\tau_{n} \geq \theta^{-3}-C_{1} \theta^{-4}=\theta^{-3}\left(1-C_{1} \theta^{-1}\right) \geq(2 / 3) \tau_{0},
$$

if $\theta$ is also sufficiently large. Moreover the inequality

$$
\left|\Lambda_{1}+\phi_{n}^{\tilde{n}}\right|+\left|X-X^{\sim}\right| \leq \tau_{n}
$$

implies that

$$
\left|\Lambda_{1}+\phi_{n-1}^{\tilde{n}}\right|+\left|X-X^{\sim}\right| \leq \tau_{n}-\left\|w_{n}^{\tilde{n}}\right\|_{0}=\tau_{n-1} .
$$

Therefore $\Omega_{n-1}\left(\tau_{n-1}\right)$ includes $\Omega_{n}\left(\tau_{n}\right)$. Since

$$
\left\|\phi_{\tilde{n}}^{\tilde{n}}\right\|_{0} \leq \tau_{0} / 3<2 \tau_{0} / 3 \leq \tau_{n}
$$

if $\theta$ is sufficiently large, the point $\left(x_{0}^{\sim}, X^{\sim}\right)$ is included in the interior of intersection of $\Omega_{n}\left(\tau_{n}\right)$ for all $n$.

Lemma 2.6. The error terms $f_{n}$ vanish absolutely on $\Omega_{n}\left(\tau_{n}\right)$, respectively. Therefore they and their limit $f_{+\infty}$ vanish on $\Omega_{+\infty}\left(\tau_{+\infty}\right)$, where $\tau_{+\infty}$ is the limit of $\tau_{n}$ as $n$ tends to infinity. The point $\left(x_{0}^{\sim}, X^{\sim}\right)$ attaining the zero of $f$ is included in the interior of $\Omega_{+\infty}\left(\tau_{+\infty}\right)$.

Proof. $f_{0}$ vanishes on $\Omega_{0}\left(\tau_{0}\right)$ according to the discussion before Lemma 2.8. We assume that $f_{n}$ vanishes on $\Omega_{n}\left(\tau_{n}\right)$. Then $\left(\Lambda_{1}+\phi_{n}^{\tilde{n}}\right) \phi_{n}^{(2)}$ vanishes on $\Omega_{n+1}\left(\tau_{n+1}\right)$ because by definition it satisfies the equation

$$
-\left\{\xi_{0}-\left(\Lambda_{1}+\phi_{n}^{\tilde{n}}\right),\left(\Lambda_{1}+\phi_{n}^{\tilde{n}}\right) \phi_{n}^{(2)}\right\}-c_{n}^{\tilde{n}}\left(\Lambda_{1}+\phi_{n}^{\tilde{n}}\right) \phi_{n}^{(2)}=-f_{n} .
$$

Therefore $\phi_{n}^{(1)}$ vanishes there so that $f_{n+1}$ vanishes on $\Omega_{n+1}\left(\tau_{n+1}\right)$.

q. e. d.

Proof of Theorem 1. It has almost finished except for differentiability of $\phi$. Lemma 2.4 shows the existence of finitely differentiable functions $\phi, c$ and $f_{+\infty}$ such that 


$$
-\left\{\xi_{0}-\left(\Lambda_{1}+\phi\right), f-\left(\Lambda_{1}+\phi\right)^{2}\right\}-c\left[f-\left(\Lambda_{1}+\phi\right)^{2}\right]=f_{+\infty} .
$$

Lemma 2.6 means that $f_{+\infty}$ vanishes on a neighborhood of the zero points of $f$ and that $\Lambda_{1}+\phi$ vanishes at the zero points of $f$. In fact $f-\left(\Lambda_{1}+\phi\right)^{2}$ is non negative on $\Omega_{+\infty}\left(\tau_{+\infty}\right)$ because it is non negative at $\Lambda_{1}+\phi=0$. And finite number of such $\Omega_{+\infty}\left(\tau_{+\infty}\right)$ cover the zero points of $f$ such that $|\xi|=1$ because it is compact.

$$
\left(\partial / \partial x_{0}\right)\left(\Lambda_{1}+\phi\right) \neq 0
$$

follows from the fact that $\|\phi\|_{1}$ is small.

At last we note the regularity of the solution $\phi$. Once Lemma 2.4 had been obtained, the decay rate $-\alpha$ in it is easily improved without moving the parameter $\theta$.

We assume the following lemma proved later.

Lemma 2.7. There exists a constant $G$ for the limit $\phi$ such that

$$
|\xi||\nabla \phi|^{2}+|\phi|^{2} \leq C f \text {. }
$$

By virtue of Lemma $2.4, \phi-\phi_{\tilde{k}}$ satisfies that

$$
\begin{aligned}
\left\|\phi-\dot{\phi}_{\tilde{k}}\right\|_{1} & \leq\left\|\phi-\phi_{k}\right\|_{1}+\left\|\phi_{k}-\phi_{\tilde{k}}\right\|_{1} \\
& \leq C_{\delta} \theta^{\alpha-3}(\theta+k)^{1-\alpha}
\end{aligned}
$$

so that

$$
|\xi|\left|\nabla \phi_{k}\right|^{2}+\left|\phi_{k}\right|^{2} \leq 2 C f+2 C_{\delta} \theta^{1-\alpha}(\theta+k)^{1-\alpha} \theta^{2 a-6} \text {. }
$$

Therefore it yields the assumption at Proposition 2.1 that

$$
|\xi|\left|\nabla \phi_{\tilde{k}}\right|^{2}+\left|\phi_{\tilde{k}}\right|^{2} \leq C\left(f+\kappa_{k}|\xi|^{2}\right)
$$

with a constant $C$ independent of $\kappa_{k}$. Hence we are able to use the estimates of $R_{j}$ at Proposition 2.1 for any non negative integer $m$. The estimates imply the following Lemma 2.8 so that we are able to assume the convergence of $\phi_{j}$ and $d_{j}$ to $\phi$ and $c$ in $\boldsymbol{H}^{a}$ for any non negative $a$. Therefore $\phi$ and $c$ are infinitely differentiable. q.e.d.

Lemma 2.8. For any positive $\beta$, and for any non negative $a$, there exists a constant $C_{a \beta}$ such that for any non negative integer $j$

$$
\| w_{j} !_{a} \text { and }\left\|d_{j}\right\|_{a} \leq C_{a \beta}(\theta+j)^{a-\beta-1} \text {. }
$$

Proof. It suffices to prove that, if it is supposed that the inequality 
holds for a constant $\beta$, then we may replace $\beta$ by $\beta+\alpha-1$, where $\beta$ and $\beta+\alpha-1$ are not integers. In fact the inequalities are guaranteed by Lemma 2.4 in the case $\beta=\alpha$. It is possible to take the same procedure in the present case as at the proof of Lemma 2.4 by replacing $\alpha$ by $\beta$ if the influence of the constant $\theta$ is neglected. So we get the estimate for $e_{k}$ corresponding to one at 3 ) of Lemma 2.5, that is,

$$
\left\|e_{k}\right\|_{a} \leq C(\theta+k)^{a-\alpha-\beta}
$$

with a constant $C$ independent of $k$. Moreover it holds that

$$
\left\|E_{k}\right\|_{\alpha+\beta-1} \leq C
$$

so that

$$
\begin{aligned}
& \left\|\left(S_{\theta(\theta+k+1)}-S_{\theta(\theta+k)}\right)\left(h_{0}^{(0)}+E_{k}\right)\right\|_{a} \\
& \quad \leq C(\theta+k+1)^{a-\alpha-\beta}\left(\left\|h_{0}^{(0)}\right\|_{\alpha+\beta-1}+\left\|E_{k}\right\|_{\alpha+\beta-1}\right) \\
& \quad \leq C(\theta+k+1)^{a-\alpha-\beta} .
\end{aligned}
$$

Therefore we obtain that for any non negative $a$,

$$
\left\|g_{k+1}\right\|_{a} \leq C_{a}(\theta+k+1)^{a-\alpha-\beta} .
$$

Proposition 2.1 combines with the above to yield the estimates for $w_{k+1}$ and $d_{k+1}$ with the rate $a-\alpha-\beta$. q. e. d.

Proof of Lemma 2.7. It suffices to consider it at a neighborhood of the zero points of $f$. Then it holds that $\Phi(\phi)-c \Psi(\phi)=0$. This implies that

$$
\left(\Lambda_{1}+\phi\right)\left[2\left(\partial / \partial x_{0}\right)\left(\Lambda_{1}+\phi\right)+c\left(\Lambda_{1}+\phi\right)\right]=\left\{f, \Lambda_{1}+\phi\right\}+\left(\partial / \partial x_{0}\right) f+c f .
$$

Since $\left[2\left(\partial / \partial x_{0}\right)\left(\Lambda_{1}+\phi\right)+c\left(\Lambda_{1}+\phi\right)\right]$ does not vanish at the zero points of $f, \phi$ is a linear combination of $\nabla f$ and $f$. Therefore

$$
\left(\Lambda_{1}+\phi\right) 2\left(\partial / \partial x_{0}\right) \phi-\{f, \phi\}+2\left(\partial / \partial x_{0}\right)\left(\Lambda_{1}\right) \phi=L[\nabla f, f: \nabla f, f] .
$$

We differentiate it to obtain the equation for $\left(z_{0}, Z\right)=\nabla \phi$ that

$$
K^{\sim}\left(z_{0}, Z\right)+2\left(\partial / \partial x_{0}\right)\left(\Lambda_{1}+\phi\right)\left(z_{0}, Z\right)=L[\nabla f, f],
$$

where

$$
K^{\sim}\left(z_{0}, Z\right)=\left(2 \Lambda_{1 x_{0}} z_{0}-f_{x_{0} X} J Z, 2 \Lambda_{1 X} z_{0}-f_{X X} J Z\right) .
$$

The hyperbolicity (See the first part of Section 1 or 3.) yields that $K^{\sim}$ has no non zero real eigenvalue at the zero points of $f$, so that

$$
K^{\sim}+2\left(\partial / \partial x_{0}\right)\left(\Lambda_{1}+\phi\right)
$$


is invertible at a neighborhood of the zero points of $f$ because $\left(\partial / \partial x_{0}\right) \phi$ is small. Therefore we conclude that $\nabla \phi$ is a linear combination of $\nabla f$ and $f$. q.e.d.

\section{§ 3. Asymptotic Behavior of Characteristic Curves}

Let us put a vector field $\mathscr{L}$ as follows.

$$
\begin{aligned}
\mathscr{L}_{v} & =-2 \Lambda\left\{\xi_{0}-\Lambda, v\right\}+\left\{f-\Lambda^{2}, v\right\} \\
& =-2 \Lambda\left\{\xi_{0}, v\right\}+\{f, v\},
\end{aligned}
$$

where

$$
\Lambda=\Lambda_{1}+\phi
$$

We denote the flow starting from $\left(x_{0}, X\right)=\left(x_{0}, x, \xi\right)$ of the vector field $|\xi|^{-1} \mathscr{L}$ on $\boldsymbol{R}^{2 n+1}$ by $F_{t}\left(x_{0}, X\right)$. We clarify the asymptotic behavior of the flow $F_{t}\left(x_{0}, X\right)$ and its derivatives in $\left(x_{0}, X\right)$ as the parameter $t$ tends to infinity.

$$
u\left(t, x_{0}, X\right)=\left(F_{t}^{*} g\right)\left(x_{0}, X\right)=g\left(F_{t}\left(x_{0}, X\right)\right)
$$

is a solution of the equation

$$
(d / d t) u-|\xi|^{-1} \mathscr{L} u=0
$$

and

$$
\left.u\right|_{t=0}=g \text {. }
$$

By definition, $\left(y_{0}, Y\right)=\left(y_{0}, y, \eta\right)=F_{t}\left(x_{0}, X\right)$ is a solution of the differential equation,

$$
\begin{aligned}
& (d / d t) y_{0}=-2 \Lambda\left(y_{0}, Y\right)|\eta|^{-1}, \\
& (d / d t) Y=J(\partial / \partial X) f\left(y_{0}, Y\right)|\eta|^{-1}
\end{aligned}
$$

and

$$
\left.\left(y_{0}, Y\right)\right|_{t=0}=\left(x_{0}, X\right),
$$

where $J$ is the matrix such that $J(y, \eta)=(\eta,-y)$. Hence we have it for $|\eta|$ that

$$
(d / d t)|\eta|^{2}=-2\left\langle(\partial / \partial x) f\left(y_{0}, Y\right), \eta\right\rangle|\eta|^{-1}
$$

so that

$$
\left.\left.|(d / d t)| \eta\right|^{2}\left|\leq 2 \beta_{0}\left(y_{0}, Y\right)\right| \eta\right|^{2},
$$

where 


$$
\beta_{0}=\left.|(\partial / \partial x) f| ! \xi\right|^{-2}
$$

This implies that

$$
|\eta|^{2} \leq|\xi|^{2} \exp \left[2 \int_{0}^{t} F_{s}^{*} \beta_{0} d s\right]
$$

and

$$
|\xi|^{2} \leq|\eta|^{2} \exp \left[2 \int_{0}^{t} F_{s}^{*} \beta_{0} d s\right] .
$$

We now consider the variational equation of (3.2) in order to estimate the derivatives of the flow $F_{t}\left(x_{0}, X\right)$ in $\left(x_{0}, X\right)$.

$$
(d / d t) z_{0}=-2\left(\Lambda|\eta|^{-1}\right)_{x_{0}} z_{0}-2\left(\Lambda|\eta|^{-1}\right)_{X} Z+g_{0}
$$

and

$$
(d / d t) Z=J\left(f_{x_{0}}|\eta|^{-1}\right)_{X} z_{0}+J\left(f_{X}|\eta|^{-1}\right)_{X} Z+g_{1}
$$

We put it as

$$
(d / d t)\left(z_{0}, Z\right)=K\left(z_{0}, Z\right)+G .
$$

We may assume that all components of $K$ are of homogeneous order 0 in $\eta$ because they are unified the order in $\eta$ by the transformation of $\left(z_{0}, z, \zeta\right)$ to $\left(z_{0}, z,|\eta|^{-1} \zeta\right)$, where $Z=(z, \zeta)$.

Remark. Our following discussion is subject to no restriction according to such a unification of order because $(d / d t)|\eta|^{2}$ satisfies (3.3) and only results modulo $L[\nabla f, f]$ are required.

By definition, it holds that

$$
\Lambda_{x_{0}}|\eta|^{-1}=\alpha^{-1} f_{x_{0} x_{0}}|\eta|^{-1}+f_{x_{0} X}|\eta|^{-1} v_{2}+L\left[\phi_{x_{0}}, \phi, \nabla f, f\right] .
$$

On the other hand also by definition it holds at a neighborhood of the zero points $\Sigma$ of $f$ that

$$
\alpha^{-1} f_{x_{0} x_{0}}+f_{x_{0} x} v_{2}=\alpha / 2
$$

and

$$
f_{X x_{0}} \alpha^{-1}+f_{X X} v_{2}=\alpha J v_{2} .
$$

Hence $K$ is written as

$$
\begin{aligned}
& K=K_{0}+K_{0}^{\prime}, \\
& K_{0}\left(z_{0}, Z\right)=\left(K_{00} z_{0}+K_{01} Z, K_{10} z_{0}+K_{11} Z\right), \\
& K_{00}=-\alpha \sim, \quad K_{01}=-2 \alpha \sim J v_{2}, \\
& K_{10}=J \nabla_{X} \nabla_{0} f|\eta|^{-1}, K_{11}=J \nabla_{X}^{2} f|\eta|^{-1}
\end{aligned}
$$

and 


$$
K_{0}^{\prime}=L[\nabla \phi, \phi, \nabla f, f],
$$

where $\alpha^{\sim}=\alpha|\eta|^{-1},\left\langle J v_{2}, Z\right\rangle=\left\langle J v_{2},\left(z,|\eta|^{-1} \zeta\right)\right\rangle$ for any $Z=(z, \zeta)$ and the following notations for $\nabla^{k}$ are used.

Remark on Notations. $\nabla^{k}$ stands for weighted partial differential operators in the rest of this paper such that

$$
\begin{aligned}
& \nabla^{k} g\left(x_{0}, x, \xi\right) \\
& \quad=\left(|\xi|^{(|\gamma|-|\alpha|-|\beta|) / 2}\left(\partial / \partial x_{0}\right)^{\alpha}(\partial / \partial x)^{\beta}(\partial / \partial \xi)^{\gamma} g\right)_{|\alpha|+|\beta|+|\gamma|=k} .
\end{aligned}
$$

Therefore

$$
\begin{aligned}
& \nabla_{0} g=|\xi|^{-1 / 2}\left(\partial / \partial x_{0}\right) g, \\
& \nabla_{X} g=\left(|\xi|^{-1 / 2}(\partial / \partial x) g,|\xi|^{1 / 2}(\partial / \partial \xi) g\right), \\
& \nabla_{0} \nabla_{X} g=\left(|\xi|^{-1}\left(\partial / \partial x_{0}\right)(\partial / \partial x) g, \quad\left(\partial / \partial x_{0}\right)(\partial / \partial \xi) g\right)
\end{aligned}
$$

and so on.

Let us transform $K_{0}$ by means of $M_{0}$ that

$$
M_{0}\left(z_{0}, Z\right)=\left(z_{0}, \alpha \sim v_{2}^{\sim} z_{0}+Z\right) \text {. }
$$

Then

$$
K_{1}=M_{0}^{-1} K_{0} M_{0}
$$

is that

$$
\begin{aligned}
& K_{1}\left(z_{0}, Z\right) \\
& \quad=\left(-\alpha \sim z_{0}-2 \alpha \sim\left\langle J v_{2} \tilde{2}, Z\right\rangle,\left(J \nabla_{X}^{2} f|\eta|^{-1}+2 \alpha^{2 v_{2}} \otimes J v_{2}\right) Z\right) .
\end{aligned}
$$

Therefore $\Theta=M_{0}^{-1}\left(z_{0}, Z\right)$ satisfies that

$$
(d / d t) \Theta=\left(K_{1}+K_{1}^{\prime}\right) \Theta+G^{\sim}
$$

and

$$
K_{1}^{\prime}=L[\nabla \phi, \phi, \nabla f, f]
$$

because

$$
(d / d t) M_{0}=L[\nabla \phi, \phi, \nabla f, f] .
$$

Let us put

$$
H_{0}=-J \nabla_{X}^{2} f|\eta|^{-1} J-2 \alpha^{\sim 2} v_{2}^{\tilde{2}} \otimes v_{2}^{\tilde{2}} .
$$

$H_{0}$ is non negative at the zero points $\Sigma$ of $f$ because $\nabla^{2} p_{2}$ is hyperbolic. For $\Theta=\left(\theta_{0}, \theta\right)$, it holds that

$$
(d / d t) \theta_{0}=-\alpha \sim \theta_{0}-2 \alpha \sim\left\langle J v_{2}^{\tilde{2}}, \theta\right\rangle+L[\nabla \phi, \phi, \nabla f, f] \Theta+g_{0}^{\tilde{a}}
$$


and

$$
(d / d t) \theta=J H_{0} \theta+L[\nabla \phi, \phi, \nabla f, f] \Theta+g_{1} .
$$

Let us next consider $H_{\delta}=H_{0}+2 \delta$ such that

$$
0<\delta \leq H_{\delta} \leq(C+2 \delta) \leq C^{\prime} .
$$

Then it holds that

$$
(C+2 \delta)^{-1} \leq H_{\delta}^{-1} \leq \delta^{-1} .
$$

The new inner product defined by

$$
(u, v)_{\delta} \equiv\left(H_{\delta}^{-1} u, v\right)
$$

has the relation with the canonical one as

$$
(C+2 \delta)^{-1}|u|^{2} \leq|u|_{\delta}^{2} \leq \delta^{-1}|u|^{2}
$$

and for any matrix $B$, its bound is estimated as

$$
\left.\left|B u_{i \delta}^{\prime 2} \leq \delta^{-1}(C+2 \delta)\right||B|\right|^{2}|u|_{\delta}^{2}
$$

and

$$
\left|(B u, u)_{\delta}\right| \leq \delta^{-1 / 2}(C+2 \delta)^{1 / 2}|| B|||u|_{\delta}^{2}
$$

The equality

$$
H_{\delta}^{-1} H_{0}=I-2 \delta H_{\delta}^{-1}
$$

yields

$$
\operatorname{Re}\left(H_{0} J u, u\right)_{\delta}=2 \delta \operatorname{Re}(J u, u)_{\delta} .
$$

Therefore if we restrict $\delta$ as it is less than a fixed constant, then we obtain

$$
\begin{aligned}
& \left|\operatorname{Re}\left(H_{0} J u, u\right)_{\delta}\right| \\
& \quad \leq 2 \delta^{1 / 2}(C+2 \delta)^{1 / 2}|u|_{\delta}^{2} \\
& \quad \leq C \delta^{1 / 2}|u|_{\delta}^{2} .
\end{aligned}
$$

On the other hand, the equality

$$
(d / d t)|u|_{\delta}^{2}=\left(H_{\delta}^{-1}\left((d / d t) H_{\delta}\right) H_{\delta}^{-1} u, u\right)
$$

implies

$$
\left.\left.|(d / d t)| u\right|_{\delta} ^{2}\left|\leq C(\delta) \beta\left(y_{0}, Y\right) \delta^{-1}\right| u\right|_{\delta} ^{2}
$$

for $u$ independent of $t$, where

$$
\beta\left(x_{0}, X\right)=|\nabla f||\xi|^{-3 / 2}+f|\xi|^{-2}+|\nabla \phi||\xi|^{-1 / 2}+|\phi||\xi|^{-1} .
$$

Therefore the solution $w$ of the equation 


$$
(d / d t) w=H_{0} J w+g_{1}
$$

is estimated as

$$
(d / d t)|w|_{\delta}^{2} \leq\left[C \delta^{1 / 2}+C(\delta) \beta\left(y_{0}, Y\right) \delta^{-1}\right]|w|_{\delta}^{2}+|w|_{\delta}\left|g_{1}\right|_{\delta}
$$

For the solution $w_{0}$, of the equation

$$
(d / d t) w_{0}^{\prime}=-\alpha \sim \ell_{0}^{\prime}+2 \alpha \sim\left\langle v_{2}, w^{\prime}\right\rangle+g_{0},
$$

it holds that

$$
(d / d t)\left|w_{0}\right|^{2} \leq-\alpha \sim\left|w_{0}\right|^{2}+C\left|w^{\prime}\right|_{\delta}\left|w_{0}\right|+\left|g_{0}\right|\left|w_{0}\right| .
$$

Hence it holds for $\left(w_{0}, w\right)$ that

$$
(d / d t)\left|w_{0}\right| \leq-\alpha^{\sim}\left|w_{0}\right|+C|w|_{\delta}+\left|g_{0}\right|
$$

and

$$
(d / d t)|w|_{\delta} \leq\left[C \delta^{1 / 2}+C(\delta) \beta\left(y_{0}, Y\right) \delta^{-1}\right]|w|_{\delta}+\left|g_{1}\right|_{\delta}
$$

because $(d / d t)\left|w_{0}\right|$ and $(d / d t)|w|_{\delta}$ are equal to zero almost everywhere on $\left\{\left|w_{0}\right|=0\right\}$ and on $\left\{|w|_{\delta}=0\right\}$, respectively. Meanwhile the relation (3.7) implies that

$$
|L[\nabla \phi, \phi, \nabla f, f] \Theta|_{\delta} \leq C \delta^{-1}\left(\left|\theta_{0}\right|+|\theta|_{\delta}\right) \beta\left(y_{0}, Y\right)
$$

and

$$
|L[\nabla \phi, \phi, \nabla f, f] \Theta| \leq C\left(\left|\theta_{0}\right|+|\theta|_{\delta}\right) \beta\left(y_{0}, Y\right) .
$$

The combination of (3.8) and (3.9) implies it for the solution $\Theta$ of (3.6) that

$$
(d / d t)\left|\theta_{0}\right| \leq\left(-\alpha^{\sim}+C \beta\left(y_{0}, Y\right)\right)\left|\theta_{0}\right|+C_{0}\left(1+\beta\left(y_{0}, Y\right)\right)|\theta|_{\delta}+\left|g_{0}^{\sim}\right|
$$

and

$$
\begin{aligned}
(d / d t)|\theta|_{\delta} & \leq\left[C \delta^{1 / 2}+C(\delta) \beta\left(y_{0}, Y\right) \delta^{-1}\right]|\theta|_{\delta} \\
& +C \delta^{-1} \beta\left(y_{0}, Y\right)\left|\theta_{0}\right|+\left|g_{1} \tilde{\mid}\right|_{\delta} .
\end{aligned}
$$

Let us put

$$
\gamma=\gamma\left(y_{0}, Y\right)=\left[C \delta^{1 / 2}+C(\delta) \beta\left(y_{0}, Y\right) \delta^{-1}\right]
$$

with some other large constants $C$ and $C(\delta)$. Then

$$
\omega_{0}=\left(\alpha_{0} / C_{0}\right)\left|\theta_{0}\right| \exp \left[-C \int_{0}^{t} \gamma d s\right]
$$

and

$$
\omega=|\theta|_{\delta} \exp \left[-C \int_{0}^{t} \gamma d s\right]
$$

satisfy it with another large constant $C$ independent of $\delta$ that 


$$
(d / d t) \omega_{0} \leq-\alpha_{0}(1+\gamma) \omega_{0}+\alpha_{0}(1+\gamma) \omega+g_{0}^{\prime}
$$

and

$$
(d / d t) \omega \leq-\gamma \omega+\gamma \omega_{0}+g_{1}^{\prime},
$$

where $\alpha_{0}$ is a constant such that

$$
\begin{gathered}
0<\alpha_{0} \leq \alpha^{\sim}, \\
g_{0}^{\prime}=\left(\alpha_{0} / C_{0}\right)\left|g_{0}^{\sim}\right| \exp \left[-C \int_{0}^{t} \gamma d s\right]
\end{gathered}
$$

and

$$
g_{1}^{\prime}=\left|g_{1}^{\tilde{1}}\right|_{\delta} \exp \left[-C \int_{0}^{t} \gamma d s\right] .
$$

The following lemma applies to it to yield

$$
\omega_{0}(t)+\omega(t) \leq 2\left(\omega_{0}(0)+\omega(0)+\int_{0}^{t}\left(g_{0}^{\prime}+g_{1}^{\prime}\right) d s\right) .
$$

Lemma 3.1. Let $u_{j}(j=1, \cdots, \nu)$ real valued functions in $t$ be absolutely continuous and satisfy almost everywhere that

$$
(d / d t) u_{j} \leq f_{j}(t, u)+h_{j}(t),
$$

where the functions $f_{j}$ satisfy that

$$
f_{j}(t, u) \leq 0
$$

if

$$
u_{j}(t) \geq u_{k}(t)
$$

for all $k$, and where $h_{j}$ are measurable functions in $t$. Then $u_{j}$ are estimated as

$$
u_{j}(t) \leq \max _{k}\left(u_{k}(s)\right)+\int_{s}^{t} \max _{k}\left(h_{k}(\sigma)\right) d \sigma
$$

for $t \geq s$. Especially if all of $u_{j}$ and $h_{j}$ are non negative, then it holds for $t \geq s$ that

$$
\sum_{j=1}^{\nu} u_{j}(t) \leq \nu\left[\sum_{j=1}^{\nu} u_{j}(s)+\int_{s}^{t} \sum_{j=1}^{\nu} h_{j}(\sigma) d \sigma\right]
$$

Therefore we get

$$
\begin{aligned}
\left|\theta_{0}(t)\right|+|\theta(t)|_{\delta} \leq & C\left(\left|\theta_{0}(0)\right|+|\theta(0)|_{\delta}\right) \exp \left[C \int_{0}^{t} \gamma d s\right] \\
& +C \int_{0}^{t}\left(\left|g_{0} \tilde{\mid}\right|+\left|g_{1}\right|_{\delta}\right) \exp \left[C \int_{s}^{t} \gamma d \sigma\right] d s .
\end{aligned}
$$

This concludes for a solution of (3.4) that 


$$
\begin{aligned}
& \left|z_{0}(t)\right|+|Z(t)| \\
& \leq C(\delta)\left\{\left(\left|z_{0}(0)\right|+|Z(0)|\right) \exp \left[C \int_{0}^{t} \gamma d s\right]+\int_{0}^{t}|G| \exp \left[C \int_{s}^{t} \gamma d \sigma\right] d s\right\}
\end{aligned}
$$

with a constant $C(\delta)$ depending on $\delta$, especially on the minimum of $\delta$.

Since $G=0$ in the case that $\left(z_{0}, Z\right)$ is one of the first derivatives $\Xi^{(1)}$ of $\left(y_{0}, Y\right)$ such that

$$
\Xi^{(1)}=\left(|\eta|^{1 / 2} \nabla y_{0}, \quad|\eta|^{1 / 2} \nabla y, \quad|\eta|^{-1 / 2} \nabla \eta\right),
$$

the inequality (3.10) gives us

$$
\left|\Xi^{(1)}(t)\right| \leq \Omega_{1}(\delta) \exp \left[C \int_{0}^{l} \gamma d s\right] .
$$

In general it turns out that

$$
\left|\Xi^{(m)}\right| \leq \Omega_{m}(\delta)\left(\|\phi\|_{m}+1\right) \exp \left[C_{m} \int_{0}^{t} \gamma d s\right],
$$

for the derivatives $\Xi^{(m)}$ of order $m$ such that

$$
\Xi^{(m)}=\left(|\eta|^{m / 2} \nabla^{m} y_{0}, \quad|\eta|^{m / 2} \nabla^{m} y, \quad|\eta|^{-m / 2} \nabla^{m} \eta\right),
$$

where $\Omega_{m}$ is some constant, which may depend on $\delta$ but is independent of $\phi$. This fact is proved by induction in $m$ and by means of Lemma 2.3.

We assume that the statement (3.11) is valid if $k \leq m-1$. The inhomogeneous term $G_{m}\left(\Xi^{l}\right)$ of (3.4) is described as

$$
G_{m}\left(\Xi^{l}\right)=\sum A_{l} \Pi_{j=1}^{\nu} \Xi^{(l(j))},
$$

where $\sum_{j=1}^{\nu} l(j) \leq m-1$ and $A_{l}$ are linear functions of $\left(\nabla^{j} \phi\right)\left(y_{0}, Y\right)$ $(j \leq \nu)$, which satisfy, therefore,

$$
\left|A_{l}\right| \leq C\left(\|\phi\|_{\nu}+1\right) .
$$

Hence each monomial is estimated by the inequalities (3.11) provided by the assumption as

$$
\begin{aligned}
& \left|A_{l} \Pi_{j=1}^{\nu} \Xi^{(l(j))}\right| \\
& \quad \leq C\left|A_{l}\right| \Pi_{j=1}^{\nu}\left|\Xi^{(l(j))}\right| \\
& \quad \leq C\left(\|\phi\|_{\nu}+1\right) \Pi_{j=1}^{\nu}\left(i \mid \phi \|_{l(j)}+1\right) \Omega_{\iota} \exp \left[G_{l} \int_{0}^{t} \gamma d s\right] .
\end{aligned}
$$

Lemma 2 applies to this in the case that

$$
\lambda_{0}=(\nu-1) /(m-1)
$$

and

$$
\lambda_{j}=\left(l^{\prime}(j)-1\right) /(m-1),
$$

where $l^{\prime}(j)$ are integers such that $l(j) \leq l^{\prime}(j)$ and $\sum_{j=1}^{\nu} l^{\prime}(j)=m$, and 
that $a(i, j)=m$ if $i=j$ and $a(i, j)=1$ if $i \neq j$. Then

$$
\begin{aligned}
& \sum_{j=0}^{\nu} \lambda_{j}=1, \\
& a(i)=\sum_{j=0}^{\nu} a(i, j) \lambda_{j}=\nu \quad \text { if } i=0,
\end{aligned}
$$

and

$$
a(i)=l^{\prime}(i) \quad \text { if } i \geq 1 \text {. }
$$

We obtain

$$
\begin{aligned}
& \|\phi\|_{\nu} \Pi_{j=1}^{\nu}\|\phi\|_{l(j)} \leq C\|\phi\|_{\nu} \Pi_{j=1}^{\nu}\|\phi\|_{l^{\prime}(j)} \\
& \quad \leq C\|\phi\|_{m}\|\phi\|_{1}^{m-1} .
\end{aligned}
$$

According to the uniform boundedness of $\|\phi\|_{1}, G_{m}$ is bounded as

$$
\left|G_{m}\right| \leq \Omega_{m}\left(\|\phi\|_{m}+1\right) \exp \left[G_{m} \int_{0}^{t} \gamma d s\right]
$$

Inserting (3.12) to (3.10), we obtain (3.11) for $\Xi^{(m)}$.

We next treat the estimates of $|\eta|^{m / 2-l} \nabla^{m}\left[g\left(y_{0}, Y\right)\right](m \geq 1)$ for an infinitely differentiable homogeneous function $g\left(x_{0}, X\right)$ of order $l$. $|\eta|^{m / 2-l} \nabla^{m}\left[g\left(y_{0}, Y\right)\right]$ consists of polynomials in $\Xi^{(k)}(1 \leq k \leq m)$ with coefficients which are linear in $\left(V^{k} g\right)\left(y_{0}, Y\right)(1 \leq k \leq m)$, namely, the type of its monomials is

$$
|\eta|^{k / 2-l}\left(\nabla^{k} g\right)\left(y_{0}, Y\right) \Pi_{j=1}^{k} \Xi^{(k(j))},
$$

where $1 \leq k \leq m$ and $\sum_{j=1}^{k} k(j)=m$. Since $\Xi^{(k(j))}$ are bounded by

$$
\Omega\left(\|\phi\|_{k(j)}+1\right) \exp \left[C \int_{0}^{t} \gamma d s\right]
$$

it must be estimated as

$$
\begin{aligned}
& \left.|| \eta\right|^{k / 2-l}\left(\nabla^{k} g\right)\left(y_{0}, Y\right) \Pi_{j=1}^{k} \Xi^{(k(j))} \mid \\
& \quad \leq C\|g\|_{k} \Pi_{j=1}^{k}\left(\|\phi\|_{k(j)}+1\right) \exp \left[C \int_{0}^{t} \gamma d s\right] .
\end{aligned}
$$

Lemma 2 applies again to these so that they have a common bound

$$
C\left(\|g\|_{m}+\|g\|_{0}\left(\|\phi\|_{m+1}+1\right)\right) \exp \left[C \int_{0}^{t} \gamma d s\right]
$$

with respect to $k(1 \leq k \leq m)$. It is shown that

$$
\begin{aligned}
& \left.|| \eta\right|^{m / 2-l} \nabla^{m}\left[g\left(y_{0}, Y\right)\right] \mid \\
& \quad \leq C\left(\|g\|_{m}+\|g\|_{0}\left(\|\phi\|_{m+1}+1\right)\right) \exp \left[C \int_{0}^{t} \gamma d s\right] .
\end{aligned}
$$

Let us consider another function $\Gamma\left(t, x_{0}, X\right)$ defined by two homogeneous functions $\Gamma_{0}$ and $\Gamma_{1}$ of order 0 such that 


$$
\Gamma\left(t, x_{0}, X\right)=\Gamma_{0}\left(x_{0}, X\right)+\Gamma_{1}\left(x_{0}, X\right) t
$$

and $\left\|\Gamma_{j}\right\|_{0}(j=0,1)$ are uniformly bounded by a constant. In the same reason as with respect to $|\eta|^{m / 2-l} \nabla^{m}\left[g\left(y_{0}, Y\right)\right]$,

is bounded by

$$
|\eta|^{m / 2} \nabla^{m} \int_{0}^{t} \Gamma\left(t-s, y_{0}(s), Y(s)\right) d s
$$

$$
C\left(\|\Gamma\|_{m}+\|\Gamma\|_{0}\left(\|\phi\|_{m+1}+1\right)\right) \int_{0}^{t}(1+t-s) \exp \left[C \int_{0}^{s} \gamma d \sigma\right] d s,
$$

where $i \mid \Gamma \|_{j}$ means $\left\|\Gamma_{0}\right\|_{j}+i \mid \Gamma_{1} \|_{j}$. It may replace to

$$
C\left(\|\Gamma\|_{m}+\|\Gamma\|_{0}\left(\|\phi\|_{m+1}+1\right)\right) \exp \left[C \int_{0}^{t} \gamma d s\right]
$$

because the minimum of $\gamma$ is positive. Since the similar argument may apply to

$$
|\eta|^{m / 2-l} \nabla^{m}\left[g\left(y_{0}, Y\right) \exp \left[-\int_{0}^{t} \Gamma\left(t-s, y_{0}(s), Y(s)\right) d s\right]\right]
$$

its bound is given by the superior of

$$
\begin{aligned}
& C\left(\|g\|_{k}+\|g\|_{0}\left(\|\phi\|_{k+1}+1\right)\right) \Pi_{j=1}^{l}\left(\|\Gamma\|_{k(j)}+\|\Gamma\|_{0}\left(\|\phi\|_{k(j)+1}+1\right)\right) \\
& \quad \times \exp \left[-\int_{0}^{t}\left(\Gamma_{t}-C \gamma\right) d s\right],
\end{aligned}
$$

where $k+\sum_{j=1}^{l} k(j)=m$ and $\Gamma_{t}=\Gamma\left(t-s, y_{0}(s), Y(s)\right)$.

According to the assumption that $\|\Gamma\|_{0}$ is bounded by a constant, the application of Lemma 2 to them gives again them a common bound such that

$$
C\left[\|g\|_{m}+\|g\|_{0}\left(\|\dot{\phi}\|_{m+1}+\|\Gamma\|_{m}+1\right)\right] \exp \left[-\int_{0}^{t}\left(\Gamma_{t}-C \gamma\right) d s\right] .
$$

We get the above results into the proposition.

Proposition 3.1. Let $\left(y_{0}, Y\right)=F_{t}\left(x_{0}, X\right)$ be the flow defined by (3.2). Then for any infinitely differentiable homogeneous function $g$ of order $l$,

$$
|\eta|^{m / 2-l} \nabla^{m}\left\{g\left(y_{0}, Y\right) \exp \left[-\int_{0}^{t} \Gamma_{t}(s) d s\right]\right\}
$$

is bounded by

$$
C_{m \gamma}\left[\|g\|_{m}+\|g\|_{0}\left(\|\phi\|_{m+1}+\left\|_{1} \Gamma\right\|_{m}+1\right)\right] \exp \left[-\int_{0}^{t}\left(\Gamma_{t}-C_{m} \gamma\right) d s\right],
$$

where

$$
\begin{aligned}
& \gamma=\gamma\left(y_{0}, Y\right) \\
& \quad=C \delta^{1 / 2}\left(y_{0}, Y\right)+C(\tilde{\boldsymbol{o}}) \delta^{-1}\left(y_{0}, Y\right) \beta\left(y_{0}, Y\right), \\
& H_{0}+\tilde{\boldsymbol{o}}>0,
\end{aligned}
$$




$$
\begin{aligned}
& \beta\left(x_{0}, X\right)=|\nabla f||\xi|^{-3 / 2}+f|\xi|^{-2}+|\nabla \phi||\xi|^{-1 / 2}+|\phi||\xi|^{-1}, \\
& \Gamma_{t}(s)=\Gamma\left(t-s, y_{0}(s), Y(s)\right)
\end{aligned}
$$

and

$$
\Gamma\left(t, x_{0}, X\right)=\Gamma_{0}\left(x_{0}, X\right)+t \Gamma_{1}\left(x_{0}, X\right)
$$

with $\Gamma_{j}$ such that

$$
\left\|\Gamma_{j}\right\|_{0} \leq C
$$

Remark. At (3.13) taken off the weight $|\eta|^{m / 2-l}$ or put back to the usual notation of derivatives $\left(\partial / \partial x_{0}\right)^{\alpha}$ and $(\partial / \partial x)^{\alpha}$ from the one $\nabla^{\alpha}$, all of the above results remain valid by means of replacements of constants $C_{m}$ by other ones according to the estimates (3.3) of the weights $|\eta|$ and $|\eta|^{-1}$ and by means of multiplications of the powers of $|\xi|$ corresponding to their homogeneous order. Therefore (3.13) may replace to

$$
\left\|g\left(y_{0}, Y\right) \exp \left[-\int_{0}^{t} \Gamma_{t}(s) d s\right]\right\|_{m}
$$

\section{§4. Construction of Right Inverses}

At the previous section, the estimates of functions moved by the flow $F_{t}\left(x_{0}, X\right)$ have been attained by means of the function $\gamma$ consisting of $\nabla f, f, \nabla \phi$ and $\phi$. It is not enough outwardly for our purpose, namely, to prove Proposition 2.1. It requires to make sure of another property of the flow $F_{t}\left(x_{0}, X\right)$.

We check Proposition 2.2 before doing it. We consider the equation

$$
\left\{\xi_{0}-\Lambda, v\right\}+c v=h,
$$

where $c$ and $h$ are homogeneous of order 0 and $l$ in $\xi$, respectively. It is necessary in our case to clarify the dependence of two type of solutions on coefficients and data. One is solutions vanishing at $\Lambda=0$ and the other is the solution equal to $f$ at $\Lambda=0$ of the homogeneous equation. The change of variable $x_{0}$ to $t$ by means of the function $t=\Lambda\left(x_{0}, X\right)$ transposes (4.1) to the following. (The inverse map is denoted by $x_{0}=\mu(t, X)$.)

$$
(\partial / \partial t) w-a(\partial / \partial X) w+c^{\sim} w=b h^{\sim},
$$

where 


$$
\begin{aligned}
& a(t, X)=\Lambda_{x_{0}}(\mu(t, X), X)^{-1} J \Lambda_{X}(\mu(t, X), X), \\
& b(t, X)=\Lambda_{x_{0}}(\mu(t, X), X)^{-1}, \\
& c^{\sim}(t, X)=\Lambda_{x_{0}}(\mu(t, X), X)^{-1} c(\mu(t, X), X), \\
& f^{\sim}(t, X)=f(\mu(t, X), X)
\end{aligned}
$$

and

$$
h^{\sim}(t, X)=h(\mu(t, X), X) .
$$

If the flow of $(\partial / \partial t) G=-a(t, G)$ starting from $X$ at $t=s$ is denoted by $G(t, s, X)$, then the solution $w$ is given by

$$
\begin{aligned}
w= & G^{*}(t, 0) f^{\sim}(0) \exp \left[-\int_{0}^{t} G^{*}(t, \sigma) c^{\sim}(\sigma) d \sigma\right] \\
& +\int_{0}^{t}\left[G^{*}(t, s) b(s) h^{\sim}(s)\right] \exp \left[-\int_{s}^{t} G^{*}(t, \sigma) c^{\sim}(\sigma) d \sigma\right] d s,
\end{aligned}
$$

where

$$
G^{*}(t, s) g(s)=g(s, G(s, t, X)) .
$$

Therefore if we define $\phi_{0}^{\tilde{a}}$ and $\phi_{1}^{\tilde{1}}$ by

$$
\phi_{0}^{\tilde{a}}=G^{*}(t, 0) f^{\sim}(0) \exp \left[-\int_{0}^{t} G^{*}(t, \sigma) c^{\sim}(\sigma) d \sigma\right]
$$

and

$$
\phi_{1}^{\tilde{1}}=\int_{0}^{1} G^{*}(t, t s)\left(b h^{\sim}\right)(t s) \exp \left[-t \int_{s}^{1} G^{*}(t, t \sigma) c^{\sim}(t \sigma) d \sigma\right] d s,
$$

then two types of solutions are given by

$$
w_{0}=\phi_{0}^{\tilde{a}}
$$

and

$$
w_{1}=t \psi_{1}^{\tilde{1}} \text {. }
$$

The converse change of variable of $t$ to $x_{0}$ gives a solution $\phi_{0}$ of (4.1) such that

$$
\phi_{0}\left(x_{0}, X\right)=\phi_{0}\left(\Lambda\left(x_{0}, X\right), X\right) .
$$

The other one vanishing at $\Lambda=0$ is given by

$$
v_{1}=\Lambda\left(x_{0}, X\right) \phi_{1}\left(x_{0}, X\right)
$$

and

$$
\phi_{1}\left(x_{0}, X\right)=\phi_{1}^{\sim}\left(\Lambda\left(x_{0}, X\right), X\right) .
$$

If $\Lambda=\Lambda_{1}+\phi$ and if $\|\phi\|_{2}$ and $\|c\|_{1}$ have a common bound, then this process of construction yields the estimates (4.2), for the functions $\phi_{0}$ and $\phi_{1}$,

$$
\left\|\phi_{j}\right\|_{a} \leq C_{a}\left(\left\|g_{j}\right\|_{a}+\left\|g_{j}\right\|_{1}\left(\|\phi\|_{a+1}+1\right)+\left\|g_{j}\right\|_{0}\left(\|c\|_{a}+1\right)\right) \text { if } 1 \leq a,
$$


and

$$
\left\|\psi_{j}\right\|_{a} \leq C_{a}\left\|g_{j}\right\|_{a} \quad \text { if } 0 \leq a \leq 1,
$$

where $g_{0}=f$ and $g_{1}=h$, because the uniqueness of solution to (4.1) assures the homogeneity of solutions so that the local estimates in $\xi$ are extended to the global one, where the estimate (4.2) is one on a conic neighborhood of $\left\{\Lambda_{1}=0\right\}$, and $\left\|_{1} \phi\right\|_{1}$ is as small as it assures $\left(\partial / \partial x_{0}\right) \Lambda \neq 0$ there. Therefore we can conclude Proposition 2.2.

The estimates (4.2) is one for general functions $\phi$. At the application of it to our case, the functions $\phi$ have a special feature such that the supports of $\phi$ concentrate at a neighborhood of the zero points of $f$. Especially the solution $\psi_{0}$ of the homogeneous equation with the initial data $f$ at $\Lambda=0$ has an advantageous estimate. We assume that $\phi$ is a product of two functions $\rho_{3 \varepsilon}\left(\varepsilon=\theta^{-4}\right)$ and $\phi^{\prime}$,

$$
\phi=\rho_{3 \varepsilon} \phi^{\prime}
$$

such that $\rho_{3 \varepsilon}$ is used at Section 2 and $\phi^{\prime}$ is estimated as

$$
\left\|\phi^{\prime}\right\|_{a} \leq C_{a} \delta \theta^{2 a-4} \text {. }
$$

Then the vector field $a(\partial / \partial X)$ is perturbed by $\phi$ only on the support of $\rho_{3 \varepsilon}$. Especially $t=\Lambda\left(x_{0}, X\right)$ is bounded as $|t| \leq C \theta^{-2}$ on the support of $\rho_{3 \varepsilon}$. Therefore the both side bounds of Jacobian of the change of variables and the estimate for $(\partial / \partial X) G(s, t, X)$ are bounded by constants independent of $\delta$ if $\theta$ is large enough to neglect the effects of $\delta$ to the bounds.

Lemma 4.1. Let $\dot{\phi}$ be a product of two functions $\rho_{3 \varepsilon}\left(\varepsilon=\theta^{-4}\right)$ and $\phi^{\prime}$

$$
\phi=\rho_{3 \varepsilon} \phi^{\prime}
$$

such that

$$
\left\|\phi^{\prime}\right\|_{a} \leq C_{a} \delta \theta^{2 a-4} \text {. }
$$

Then two solutions $\dot{\phi}_{0}$ and $\phi_{1}$ of the equation (4.1) with $\Lambda=\Lambda_{1}+\phi$ have the estimates such that

$$
\left\|\phi_{0}\right\|_{2} \leq C_{0}\left(\|\left. f\right|_{12}\right)
$$

and

$$
\left\|\phi_{1}\right\|_{1} \leq C_{1} \mid i h \|_{1}
$$


if $\theta$ is sufficiently large, where the constants $C_{0}\left(\|f\|_{2}\right)$ and $C_{1}$ are independent of the parameter $\delta$.

Proof. The proof with respect to $\phi_{1}$ is clear from the discussion before the lemma. The parts of the estimate of $(\partial / \partial X)^{2} G(0, t, X)$ affected by the perturbation $\phi$ are in the case where the path $G(s, t, X) 0 \leq s \leq t$ touches the support of $\rho_{3 \varepsilon}$. $\left((\partial / \partial X) f^{\sim}\right)(0, G(0, t, X))$ in this case is bounded by $C \theta^{-2}$ so that

$$
\left((\partial / \partial X) f^{\sim}\right)(0, G(0, t, X))(\partial / \partial X)^{2} G(0, t, X)
$$

is as small as necessary if $\theta$ is large. The effect at the change of variable by $\phi$ is also on the support of $\rho_{3 \varepsilon} . \quad(\partial / \partial X) f^{\sim}(t, X)$ and the difference

$$
(\partial / \partial X) \psi_{0}^{\tilde{0}}(0, X)-(\partial / \partial X) \psi_{0}^{\tilde{0}}(t, X)
$$

is bounded by $C \theta^{-2}$ on such a point $(t, X)$. Therefore it is possible to take the bound of $\left\|\psi_{0}\right\|_{2}$ independent of $\delta$.

q. e. d.

Another characteristic of the flow $F_{t}\left(x_{0}, X\right)$ is found out in the behavior of $\Lambda\left(y_{0}, Y\right)$ as the parameter $t$ tends to infinity. $\Lambda\left(y_{0}, Y\right)$ should decrease in exponential order if the function $f$ were identically equal to zero. Therefore it is supposed to remain small if $f$ and its derivatives are small.

Let $\psi_{0}$ and $\psi_{1}$ stand for the following ones.

$$
f-\Lambda^{2}=\psi_{0}+2 \Lambda \psi_{1}
$$

and

$$
\left\{\xi_{0}-\Lambda, \phi_{0}\right\}+c \psi_{0}=0 .
$$

Then the vector field $\mathscr{L}$ is written as

$$
\begin{aligned}
\mathscr{L}_{v} & =-2 \Lambda\left\{\xi_{0}, v\right\}+\{f, v\} \\
& =-2 \Lambda\left\{\xi_{0}-\Lambda, v\right\}+\left\{\psi_{0}+2 \Lambda \psi_{1}, v\right\} .
\end{aligned}
$$

Therefore it holds for $\Lambda, \psi_{0}$ and $\psi_{1}$ that

$$
\begin{aligned}
\mathscr{L} \Lambda & =-2 \Lambda\left\{\xi_{0}-\psi_{1}, \Lambda\right\}+\left\{\psi_{0}, \Lambda\right\}, \\
\mathscr{L} \psi_{0} & =-2 \Lambda\left\{\xi_{0}-\Lambda, \psi_{0}\right\}+2\left\{\Lambda \psi_{1}, \psi_{0}\right\} \\
& =2 \Lambda c \psi_{0}+2 \Lambda\left\{\psi_{1}, \phi_{0}\right\}+2 \psi_{1}\left\{\Lambda, \phi_{0}\right\}
\end{aligned}
$$

and

$$
\mathscr{L} \psi_{1}=-2 \Lambda\left\{\xi_{0}-\Lambda, \dot{\psi}_{1}\right\}+\left\{\psi_{0}, \psi_{1}\right\}+2 \psi_{1}\left\{\Lambda, \phi_{1}\right\}
$$


These imply the inequalities that

$$
\begin{aligned}
& \Lambda \mathscr{L} \Lambda \leq-2 \Lambda^{2}\left(\Lambda_{x_{0}}-\left|\nabla \psi_{1}\right||\nabla \Lambda|\right)+\left|\nabla \psi_{0}\right||\nabla \Lambda||\Lambda| . \\
& -\mathscr{L} \psi_{0} \leq 2|\Lambda c| \phi_{0}+2|\Lambda|\left|\nabla \psi_{0}\right|\left|\nabla \psi_{1}\right|+\left|\psi_{1}\right||\nabla \Lambda|\left|\nabla \psi_{0}\right|
\end{aligned}
$$

and

$$
\begin{aligned}
-\phi_{1} \mathscr{L} \psi_{1} \leq 2|\Lambda|\left(\left|\psi_{1 x_{0}}\right|+|\nabla \Lambda|\left|\nabla \psi_{1}\right|\right)\left|\psi_{1}\right| \\
+\left|\nabla \psi_{0}\right|\left|\nabla \psi_{1}\right|\left|\psi_{1}\right|+2 \psi_{1}^{2}|\nabla \Lambda|\left|\nabla \psi_{1}\right| .
\end{aligned}
$$

Since the non negativity of the function $\psi_{0}$ implies that $\nabla \psi_{0}$ is bounded as

$$
\left|\nabla \psi_{0}\right|^{2} \leq \psi_{0}|| \psi_{0} \|_{2}
$$

with a maximum norm $\|\cdot\|_{2}$ up to the second derivatives, so it holds that

$$
\begin{aligned}
& \Lambda \mathscr{L} \Lambda \leq-2\left(\Lambda_{x_{0}}-\left|\nabla \psi_{1}\right||\nabla \Lambda|-\varepsilon_{1}|\nabla \Lambda|^{2}\right) \Lambda^{2}+\left(8 \varepsilon_{1}\right)^{-1}|| \psi_{0} \mid \|_{2} \psi_{0}, \\
& -\alpha \mathscr{L} \psi_{0} \leq 2\left(\alpha|\Lambda c|+\left\|\psi_{0}\right\|_{2}\right) \psi_{0}+\alpha^{2}\left|\nabla \psi_{1}\right|^{2} \Lambda^{2}+(\alpha / 2)^{2}|\nabla \Lambda|^{2}\left|\psi_{1}\right|^{2}
\end{aligned}
$$

and

$$
\begin{aligned}
-\beta \psi_{1} \mathscr{L} \psi_{1} \leq & {\left[3 \beta\left(\left|\psi_{1 x_{0}}\right|+|\nabla \Lambda|\left|\nabla \psi_{1}\right|\right)+\left(\beta\left|\nabla \psi_{1}\right|\right)^{2}\right]\left|\psi_{1}\right|^{2} } \\
& +\beta\left(\left|\psi_{1 x_{0}}\right|+|\nabla \Lambda|\left|\nabla \psi_{1}\right|\right) \Lambda^{2}+4^{-1}|| \psi_{0} \|_{2} \psi_{0}
\end{aligned}
$$

for positive numbers $\varepsilon_{1}, \alpha$ and $\beta$. Therefore it is obtained that

$$
\begin{aligned}
& \Lambda \mathscr{L} \Lambda-2^{-1} \alpha \mathscr{L} \psi_{0}-\beta \psi_{1} \mathscr{L} \phi_{1} \\
& \quad \leq-2\left(\Lambda_{x_{0}}-Z_{0}\right) \Lambda^{2}+\left(\alpha|\Lambda c|+Z_{1}\right) \psi_{0}+Z_{2} \psi_{1}^{2},
\end{aligned}
$$

where

$$
\begin{aligned}
Z_{0}= & \left|\nabla \psi_{1}\right||\nabla \Lambda|+\varepsilon_{1}|\nabla \Lambda|^{2}+(\alpha / 2)^{2}\left|\nabla \psi_{1}\right|^{2} \\
& +(\beta / 2)\left(\left|\psi_{1 x_{0}}\right|+|\nabla \Lambda|\left|\nabla \psi_{1}\right|\right), \\
Z_{1}= & \left(5 / 4+\left(8 \varepsilon_{1}\right)^{-1}\right)\left\|\psi_{0}\right\|_{2}
\end{aligned}
$$

and

$$
Z_{2}=\left[3 \beta\left(\left|\psi_{1 x_{0}}\right|+|\nabla \Lambda|\left|\nabla \psi_{1}\right|\right)+\left(\beta\left|\nabla \psi_{1}\right|\right)^{2}+2^{-1}(\alpha / 2)^{2}|\nabla \Lambda|^{2}\right] .
$$

If $\varepsilon_{1}$ and $\left\|\nabla \psi_{1}\right\|$ are sufficiently small and if $|\Lambda||\xi|^{-1} \leq \varepsilon_{0}$ for some positive $\varepsilon_{0}$, then

$$
2\left(\Lambda_{x_{0}}-Z_{0}\right) \geq K|\xi|>0
$$

where

$$
K=\inf \left\{\Lambda_{x_{0}}|\xi|^{-1}\right\}>0 .
$$

This implies that 


$$
\begin{aligned}
\Lambda \mathscr{L} \Lambda & -2^{-1} \alpha \mathscr{L} \phi_{0}-\beta \phi_{1} \mathscr{L} \phi_{1} \\
\leq & -K|\xi|\left(\Lambda^{2}-\alpha \phi_{0}-\beta \psi_{1}^{2}\right)+\left(\alpha(|\Lambda c|-K|\xi|)+Z_{1}\right) \psi_{0} \\
& +\left(Z_{2}-\beta K|\xi|\right) \psi_{1}^{2} .
\end{aligned}
$$

If it is here assumed that

$$
|\Lambda c|-K|\xi| \leq-2^{-1} K|\xi|
$$

and that $\alpha$ and $\beta$ are chosen properly and large (naturally, ||$\psi_{1} \|$ should be taken small depending on $\alpha$ and $\beta$ ), then it holds that

$$
\Lambda \mathscr{L} \Lambda-2^{-1} \alpha \mathscr{L} \psi_{0}+\beta \psi_{1} \mathscr{L} \psi_{1} \leq-K|\xi|\left(\Lambda^{2}-\alpha \psi_{0}-\beta \psi_{1}^{2}\right) \text {. }
$$

Since it holds by definition that

$$
\begin{aligned}
& (d / d t)\left[\left(\Lambda^{2}-\alpha \phi_{0}-\beta \phi_{1}^{2}\right)\left(y_{0}, Y\right)\right] \\
& \quad=|\xi|^{-1} \mathscr{L}\left[\left(\Lambda^{2}-\alpha \psi_{0}-\beta \phi_{1}^{2}\right)\left(y_{0}, Y\right)\right] \\
& \quad=\left[|\xi|^{-1} \mathscr{L}\left(\Lambda^{2}-\alpha \psi_{0}-\beta \psi_{1}^{2}\right)\right]\left(y_{0}, Y\right),
\end{aligned}
$$

it is concluded that there exist positive constants $\alpha$ and $\beta$ such that

$$
(d / d t)\left(\Lambda^{2}-\alpha \psi_{0}-\beta \psi_{1}^{2}\right) \leq-2 K\left(\Lambda^{2}-\alpha \phi_{0}-\beta \psi_{1}^{2}\right)
$$

if $\quad(\Lambda c)\left(y_{0}, Y\right)\left|\leq 2^{-1} K\right| \eta \mid$ and $\left|\Lambda\left(y_{0}, Y\right)\right| \leq \varepsilon_{0}|\eta|$, and if $\left\|\nabla \phi_{1}\right\|$ are sufficiently small, where $K$ is given by

$$
K=\inf \left\{\Lambda_{x_{0}}|\xi|^{-1}\right\}>0 \text {. }
$$

The above inequality means that

$$
\left(\Lambda^{2}-\alpha \phi_{0}-\beta \phi_{1}^{2}\right)\left(y_{0}, Y\right) \leq\left(\Lambda^{2}-\alpha \psi_{0}-\beta \psi_{1}^{2}\right)\left(x_{0}, X\right) \exp [-2 K t],
$$

so that the following lemma is concluded.

Lemma 4.2. Let $\phi_{0}$ and $\phi_{1}$ defined by (4.3). There exist positive constant $\alpha$ and $\beta$ such that

$$
\Lambda^{2}\left(y_{0}, Y\right) \leq \alpha \psi_{0}\left(y_{0}, Y\right)+\beta \psi_{1}^{2}\left(y_{0}, Y\right)+\Lambda^{2}\left(x_{\uparrow}, X\right) \exp [-2 K t]
$$

if

$$
\left|(\Lambda c)\left(y_{0}, Y\right)\right| \leq 2^{-1} K|\eta|
$$

and

$$
\left|\Lambda\left(y_{0}, Y\right)\right| \leq \varepsilon_{0}|\eta|,
$$

and if $\left\|\nabla \phi_{1}\right\|$ are sufficiently small, where $K$ is given by

$$
K=\inf \left\{\Lambda_{x_{0}} \mid \xi i^{-1}\right\}>0 \text {. }
$$

Remark. $\alpha, \beta$ and the bound of $\left\|\nabla \phi_{1}\right\|$ are taken uniformly as far 
as $K, \varepsilon_{0}$ and the bound of $\left\|\psi_{0}\right\|_{2}$ are fixed.

We check that the assumptions of the previous lemma are fulfilled. One of them is that $\Lambda^{2}\left(y_{0}, Y\right)|\eta|^{-2}$ is as small as $\Lambda^{2}\left(x_{0}, X\right)|\xi|^{-2}$. This is proved as well as the previous one if the parameter $\nu$ of $f_{\nu}$ introduced at Section 1 is put small. Since

$$
\Lambda=\Lambda_{1}+\phi
$$

and

$$
\begin{aligned}
\mathscr{L} \Lambda & =-2 \Lambda\left(\partial / \partial x_{0}\right) \Lambda+\left\{f_{\nu}, \Lambda\right\} \\
& =-2 \Lambda\left[\left(\partial / \partial x_{0}\right) \Lambda-\left\{\Lambda_{1}, \Lambda\right\}\right]-2 \phi\left\{\Lambda_{1}, \Lambda\right\}+\left\{f_{\nu}-\Lambda_{1}^{2}, \Lambda\right\},
\end{aligned}
$$

it holds that

$$
\begin{aligned}
& (\Lambda \mathscr{L} \Lambda)\left(y_{0}, Y\right) \\
& \quad \leq-2 \Lambda^{2}\left[\left(\partial / \partial x_{0}\right) \Lambda_{1}+\left(\partial / \partial x_{0}\right) \phi-\left\{\Lambda_{1}, \phi\right\}\right]+C \nu|\eta|^{2}+C\left\|\phi^{2}\right\|_{1}|\eta|^{2} .
\end{aligned}
$$

If $\Lambda^{2}\left(y_{0}, Y\right)|\eta|^{-2}$ remains less than a small positive constant $\varepsilon_{0}^{2}$ and if $\|\phi\|_{0}$ is small, then

$$
\left(\partial / \partial x_{0}\right) \Lambda_{1} \geq K|\eta|
$$

there with a positive constant $K$. Therefore if $\|\phi\|_{1}$ is restricted as small as

$$
\left|\left(\partial / \partial x_{0}\right) \phi-\left\{\Lambda_{1}, \phi\right\}\right| \leq K|\eta| / 4
$$

then it holds that

$$
(\Lambda \mathscr{L} \Lambda)\left(y_{0}, Y\right) \leq-(3 / 2) K \Lambda^{2}\left(y_{0}, Y\right)+\left.\left.C \nu\right|_{1} \eta\right|^{2}+\left\|\phi^{2}\right\|_{1}|\eta|^{2} .
$$

On the other hand by the definition of the flow $F_{t}\left(x_{0}, X\right)$,

$$
\begin{aligned}
\left.|(d / d t)| \eta\right|^{-2} \mid & \leq 2\left|\nabla f_{\nu}\right||\eta|^{-7 / 2} \\
& \leq C\left(\left|\Lambda_{1}\right||\eta|^{-1}+\nu\right)|\eta|^{-2} .
\end{aligned}
$$

Hence if $\varepsilon_{0}$ and $\|\phi\|_{0}$ are taken as small as

$$
C\left|\Lambda_{1}\right||\eta|^{-1} \leq K / 2
$$

then it holds that

$$
(d / d t)\left(\Lambda^{2}\left(y_{0}, Y\right)|\eta|^{-2}\right) \leq-K \Lambda^{2}\left(y_{0}, Y\right)|\eta|^{-2}+C \nu+\left\|\phi^{2}\right\|_{1},
$$

so that

$$
\Lambda^{2}\left(y_{0}, Y\right)|\eta|^{-2} \leq \Lambda^{2}\left(x_{0}, X\right)|\xi|^{-2} \exp [-K t]+K^{-1}\left(C \nu+\left\|\phi^{2}\right\|_{1}\right)
$$

if $\left|\Lambda\left(y_{0}, Y\right)\right||\eta|^{-1} \leq \varepsilon_{0}$ and if $\|\phi\|_{1}$ is small. This concludes the following. 
Lemma 4.3. If it holds that

$$
\begin{aligned}
& \left|\Lambda_{1}\left(x_{0}, X\right)\right||\xi|^{-1} \leq \varepsilon / 4 \\
& |\phi||\xi|^{-1} \leq \varepsilon / 4
\end{aligned}
$$

and

$$
\begin{aligned}
& K^{-1}\left\|\phi^{2}\right\|_{1} \leq \varepsilon / 4 \\
& \left(K=\inf _{\left|\Lambda_{1}\right| \leq(\varepsilon / 4)|\xi|}\left[\left(\partial / \partial x_{0}\right) \Lambda_{1}|\xi|^{-1}\right]\right)
\end{aligned}
$$

and if $\nu$ of $f_{\nu}$ is sufficiently small, then

$$
\left|\Lambda\left(y_{0}, \mathrm{Y}\right)\right||\eta|^{-1} \leq \varepsilon
$$

where $\varepsilon \leq \varepsilon_{0}$ and $\|\phi\|_{1}$ should be restricted as less than some positive constant independent of $\varepsilon$ and $\nu$. Therefore it holds for any $t$ that

$$
\left|(\Lambda c)\left(y_{0}, Y\right)\right||\eta|^{-1} \leq 2^{-1} K
$$

if $2 \varepsilon\|c\|_{0} \leq K$, that is, if the flow $\left(y_{0}, Y\right)$ starts from the sufficiently small neighborhood of $\Lambda_{1}=0$ and if $\nu$ of $f_{\nu}$ is sufficiently small. It also holds for $f_{\nu}$ under the same situations that

$$
f_{\nu}\left(y_{0}, Y\right)|\eta|^{-2} \leq 2 \varepsilon
$$

because

$$
\left|f_{\nu}-\Lambda_{1}^{2}\right| \leq C \nu^{2}|\xi|^{2}
$$

Proposition 4.1. Let $\phi$ be a product of two functions $\rho_{3 \varepsilon}\left(\varepsilon=\theta^{-4}\right)$ and $\phi^{\prime}$,

$$
\phi=\rho_{3 \varepsilon} \phi^{\prime}
$$

such that

$$
\left\|\phi^{\prime}\right\|_{a} \leq C_{a} \delta \theta^{2 a-4} \text {. }
$$

Then there exist positive constants $\alpha, \nu, \theta_{0}$ and $K$, and a neighborhood $\Omega$ of the zero points of $f$ such that, if $\theta \geq \theta_{0}$, it holds on $\Omega$ that

$$
\begin{aligned}
& f_{\nu}\left(y_{0}, Y\right)|\eta|^{-2} \\
& \quad \leq \alpha\left[\left(\psi_{0}\left(y_{0}, Y\right)+\phi_{1}^{2}\left(y_{0}, Y\right)\right)|\eta|^{-2}+\exp (-K t)\right],
\end{aligned}
$$

where $\phi_{0}$ and $\phi_{1}$ are defined by (4.3).

Proof. By the definition (4.3) of $\phi_{j}$, it holds that

$$
f \leq \phi_{0}+\phi_{1}^{2}+2 \Lambda^{2} \text {. }
$$

Therefore it suffices to show the inequality for $\Lambda^{2}|\xi|^{-2}$. This is 
assured by Lemma 4.2. In fact we suppose the results valid. Then there exist constants $\alpha$ and $K$ such that

$$
\begin{aligned}
& \Lambda^{2}\left(y_{0}, Y\right)|\eta|^{-2} \\
& \quad \leq \alpha\left[\left(\psi_{0}\left(y_{0}, Y\right)+\phi_{1}^{2}\left(y_{0}, Y\right)\right)|\eta|^{-2}+\Lambda^{2}\left(x_{0}, X\right)|\eta|^{-2} \exp (-2 K t)\right] .
\end{aligned}
$$

Here we know the inequality about $|\eta|^{-2}$ such that

$$
|\eta|^{-2} \leq|\xi|^{-2} \exp \left[2 \int_{0}^{t} F_{s}^{*} \beta_{0} d s\right] \text {. }
$$

If a neighborhood $\Omega$ of the zero points of $f$ is defined as Lemma 4.3 holds, then

$$
|\eta|^{-2} \leq|\xi|^{-2} \exp (4 \varepsilon t)
$$

Therefore we get the conclusion if $\varepsilon$ is defined as $4 \varepsilon \leq K$.

Next we note that Lemma 4.2 is valid. If the domain $\Omega$ is taken as the above, then the conditions for $\Lambda\left(y_{0}, Y\right)$ are satisfied by virtue of Lemma 4.3. The assumption for $\phi$ yields Lemma 4.1. Since $\left\|f_{\nu}\right\|_{2}$ are uniformly bounded when the parameter $\nu$ tends to zero, $\left\|\phi_{0}\right\|_{2}$ is bounded independent of $\nu$ and $\delta$. Therefore the constants $\alpha, \beta$ and the bound of $\left\|\psi_{1}\right\|_{1}$ are fixed independent of $\nu$. On the other hand it holds for

$$
\begin{aligned}
h & =\Phi(\phi)-c \Psi(\phi) \\
& =\Phi(0)-c \Psi(0)+\Phi_{1}(0) \phi-c \Psi_{1}(0) \phi+\Phi_{2}(\phi)-c \Psi_{2}(\phi)
\end{aligned}
$$

that $\|\Phi(0)-c \Psi(0)\|_{1}$ is small as $\nu$ tends to zero by the discussion at the end of Section 1 and that the others are small so that there exists $\nu$ as $\left\|\psi_{1}\right\|_{1}$ is less than the bound by virtue of Lemma 4.1.

q. e. d.

Proof of Proposition 2.1. At Proposition 3.1, we put

$$
\Gamma_{0}=2\left(\partial / \partial x_{0}\right)\left(\Lambda_{1}+\phi\right)|\xi|^{-1}+2 c\left(\Lambda_{1}+\phi\right)|\xi|^{-1}+\omega^{-1}\left(1-\rho_{\omega}\right)
$$

and

$$
\Gamma_{1}=\phi|\xi|^{-2}=\left(\phi_{0}+\phi_{1}^{2}\right)|\xi|^{-2}+\kappa
$$

Then

$$
S(t) g=g\left(y_{0}, Y\right) \exp \left[-\int_{0}^{t} \Gamma_{t}(s) d s\right]
$$

is a solution of the equation

$$
\left[(d / d t)-|\xi|^{-1} \mathscr{L}+\Gamma_{0}+\Gamma_{1} t\right] S(t) g=0
$$

and

$$
S(0) g=g \text {. }
$$


Since a solution of stationary equation is given by integral of $S(t)$ in $t$ from zero to infinity, we define $R_{j}(\phi, c)$ as

$$
R_{1}(\phi, c) g=\rho_{\omega} \int_{0}^{+\infty} S(t) d t\left(|\xi|^{-1} g\right)
$$

and

$$
R_{0}(\phi, c)=-\rho_{\omega}|\xi|^{-1} \int_{0}^{+\infty} t S(t) d t\left(|\xi|^{-1} g\right)
$$

if they exist. Then they give a right inverse, that is, outside the support of $\left(1-\rho_{\omega}\right)$, it means

$$
\left(\Phi_{1}(\phi)-c \Psi_{1}(\phi)\right) R_{1}(\phi, c) g-\phi R_{0}(\phi, c) g=g .
$$

Therefore it suffices for completing the proof to show that $R_{j}(\phi, c)$ are well defined and satisfy the estimates. To be well defined is assured if

$$
\exp \left[-\int_{0}^{t}\left(\Gamma_{t}-C_{m} \gamma\right) d s\right]
$$

is integrable in $t$. In fact it is bounded by

$$
\exp \left[-2^{-1} \kappa t^{2}+C_{m} \int_{0}^{t} \gamma d s\right]
$$

if $\omega$ is taken as $\Gamma_{0}>0$. Since $\gamma$ is a bounded function, it is integrable in $t$. The more precise considerations are necessary to get the desirable estimates. We fix an integer $m$. At first we take $\delta$ in $\gamma$ small at the zero points of $f$ as

$$
\Gamma_{0}>G_{m} \gamma+\varepsilon
$$

there if the bound of $\|\phi\|_{1}$ is small, and next $\omega$ small as

$$
\Gamma_{0}>G_{m} \gamma+\varepsilon
$$

on the whole space. This implies that

$$
\exp \left[-\int_{0}^{t}\left(\Gamma_{t}-C_{m} \gamma\right) d s\right] \leq \exp (-\varepsilon t) .
$$

Therefore the constant of the estimate at Proposition 2.1 is independent of $\phi$. We now fix $\omega$ and assume it for $\phi$ that for a constant $C_{2}^{\tilde{2}}$,

$$
|\xi||\nabla \phi|^{2}+|\phi|^{2} \leq C_{2} f+\kappa|\xi|^{2} .
$$

This implies that for any non negative $m$,

$$
C_{m} \gamma \leq C_{m 3}\left(f|\xi|^{-2}+\kappa\right) .
$$

Here $\delta$ in $\gamma$ is changed depending on $m$ but $\omega$ and the bound of 
$\|\phi\|_{1}$ keep fixed as

$$
\Gamma_{0} \geq \varepsilon>0 \text {. }
$$

According to Proposition 4.1, this is bounded as

$$
C_{m} \gamma\left(y_{0}, Y\right) \leq C_{m 4}\left[\psi\left(y_{0}, Y\right)|\eta|^{-2}+\exp (-K t)\right]
$$

on a neighborhood of the zero points of $f$ independent of $m$. Therefore it holds that

$$
-\int_{0}^{t}\left(\Gamma_{t}-C_{m} \gamma\right) d s \leq-\varepsilon t+C_{m 5}
$$

This yields the uniform constant of estimate for such $\phi$ and independent of $\psi$, especially, $\kappa$ as far as $C_{2} \sim$ is fixed if the support of $\rho_{\omega}$ is included in such a neighborhood.

q. e. d.

\section{References}

[1] Hörmander, L., The boundary problems of physical geodesy, Arch. Rat. Mech. Anal., 62 (1976), 1-52.

[2] - The Cauchy problem for differential equations with double characteristics, J. Analyse Math., 32 (1977), 118-196.

[3] Ivriú, V. Ya and Petkov, V. M., Necessary conditions for the Cauchy problem for non-strictly hyperbolic equations to be well-posed, Uspehi Mat. Nauk, 29 (1974), 3-70. (Russian Math. Surveys, 29 (1974), 1-70.)

[4] Iwasaki, N., The Cauchy problem for effectively hyperbolic equations (a special case), J. Math. Kyoto Univ., 23-3 (1983), 503-562. 